\title{
Multiscale modelling of granular materials in boundary value problems accounting for mesoscale mechanisms
}

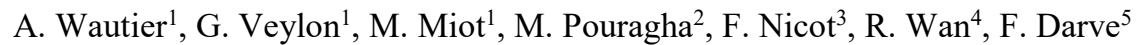

5

(1) INRAE, Unité de Recherche RECOVER, Aix-en-Provence (France)

(2) Carleton University, Civil and Environmental Engineering Dept., Ottawa, (Canada)

(3) Université Grenoble-Alpes, INRAE, Unité de Recherche ETNA, Grenoble (France)

(4) University of Calgary, Calgary (Canada)

(5) Université Grenoble-Alpes, Laboratoire Sols Solides Structures, Grenoble (France)

Corresponding author: francois.nicot@inrae.fr

\begin{abstract}
The proper solution of geotechnical boundary value problems requires robust constitutive models that can describe the mechanical behavior of geomaterials under various loading conditions, while also accounting as closely as possible for the different material scales of interest. This is even more relevant to granular media where the complexity of the mechanical behaviour is not limited to the nature of the contact law between grains, and instead originates from the multiplicity of contacts oriented along all the directions of the physical space to form distinctive mesostructures. This paper revisits the so-called H-model, which belongs to the broad family of micromechanical approaches whereby an intermediate scale (mesoscale) is explicitly introduced into the formulation. One great advantage of the model is that it can be extended by accounting for further multi-physical couplings, as for example the presence of capillary bridges between grains. This versatile model was implemented within an explicit finite difference based computational software (FLAC), and the present work demonstrates its ability to analyze engineering problems with a microstructural viewpoint, while also providing new insights in microstructural mechanisms of failure difficult to capture with standard phenomenological models.
\end{abstract}

Keywords: Granular material, Homogenization, Multiscale approach, Multislip theory, Microstructure, Mesoscopic scale, Constitutive modeling, H-model, Boundary value problems 


\section{Introduction}

There has been a marked interest in granular matter in the last few years in materials engineering, including soil mechanics, among others. Granular materials are encountered in a variety of engineering applications such as pharmaceutical engineering, food particle storage, and civil engineering. They have captured the attention of researchers due to the wide spectrum of emergent features they exhibit (Tordesillas, 2007; Tordesillas and Muthuswamy, 2009). Many peculiar properties that distinguish granular materials from solids and fluids at the macroscopic level are absent at smaller scales, namely the contact scale or even larger scales involving a few grains, which highlight the multi-scale nature of granular assembly. As salient constitutive properties of granular materials, one can list for instance: i) the incrementally nonlinear character of the constitutive response (Darve, 1990a), ii) the non-associated character of granular plasticity (Darve, 1990b; Darve et al., 1995), iii) the existence of a plastic potential and its related flow rule that can be regular or irregular (Nicot and Darve, 2007a and 2007b), and iv) the existence of a bifurcation domain in which a variety of failure modes can be encountered (Nicot and Darve, 2011a).

Historically, constitutive relations that capture these features were first proposed within a socalled phenomenological approach where models were developed in a proper, enriched mathematical framework to formulate the different observed phenomena directly at the specimen scale. Elastoplastic theories (e.g. Taylor, 1934 and 1938; Drucker and Prager, 1952; Hill, 1967a and 1967b; Rice, 1970 and 1975), generalized plasticity (Zienkiewicz and Mroz, 1984; Pastor, 1990), endochronic models (Bazant, 1978), hypoplastic theories (Kolymbas, 1991 and 1999), and incrementally nonlinear models (Darve, 1990 and 1995) belong to this class of approach. However, the accuracy of these models relies on a relatively large number of parameters that are often difficult to identify in practice and sometimes difficult to interpret from a microscale point of view (Wautier et al. 2019). The most popular of these models have been tested through a rigorous benchmark procedure, see for instance the proceedings of the workshop organized in Cleveland (Saada, 1989).

In 1938, Taylor opened the way for a new class of constitutive models for polycrystalline metals marked by multiple coexisting plastic mechanisms. He postulated that the constitutive properties could be described by relations between stress and strain components on independent planes having various orientations, thus founding the so-called multislip theory (Taylor, 1938; Batdorf and Budianski, 1949). Later, the theory was adapted for geomaterials (Zienkiewicz and Pande, 1977; Pande and Sharma, 1981 and 1982; Bazant and Gambarova, 1984). As far as 
concrete and cohesive geomaterials are concerned, the inelastic response stems mostly from damage mechanisms such as microcracking or microfracturing. The more general term "microplane model" was therefore suggested with such a fractured continuum in mind (Bazant and Oh, 1983 and 1985; Bazant, 1984). This approach lies clearly within the continuity of Hill's multislip theory (Hill, 1965, 1966 and 1967b), which is a general type of cross coupling between glide and hardening on different slip systems, as observed in metals or alloys. Microplane models belong to the broader category of multiscale approaches where the macroscopic constitutive properties emerge from the collective response of the local behaviors at the micro or mesoscopic level.

These specific aspects of multislip (or microplane) theory can be extended to granular media with the exception that, granular materials are not characterized by micro-fractures embedded in a continuous matrix, but by a disordered packing including contact planes between grains oriented along various directions (Nemat-Nasser and Mehrabadi, 1984; Nemat-Nasser, 2000). Thus, contrary to the incremental multi-linearity of the constitutive relations of metals and alloys, geomaterials are characterized by the full incremental non-linearity of their constitutive relations that originates from coarse graining of the multiple interparticle interactions along different contact orientations. The granular feature is visible through geometrical aspects (such as grain contact opening, closure or sliding), and the existence of specific units of a few grains (such as force chains (Horne, 1965; Drescher and de Josselin de Jong, 1972; Radjai et al., 1999; Tordesillas and Muthuswamy, 2009) or grain clusters (Walker and Tordesillas, 2010, Tordesillas et al., 2010)). One of the greatest advantages of multiscale approaches is that the local behavior on the contact scale can be described via simple elastic-plastic contact laws, without resorting to any sophisticated ingredients. The complexity of the constitutive behavior of granular assemblies then naturally emerges from the multiplicity of such local interactions and the evolution of the interaction network.

A thorough review of such multiscale approaches to the mechanics of granular media can be found in the seminal works of the past few decades; see for instance Nemat-Nasser and Mehrabadi, 1984; Jenkins and Strack, 1993; Mehrabadi et al., 1993; Balendran and NematNasser, 1993a and 1993b; Nemat-Nasser, 2000 and 2002.

In the continuity of microplane models (Zienkiewicz and Pande, 1977), the microdirectional model (Nicot and Darve, 2005) can be interpreted as a micromechanical application of the multislip theory to granular materials. From such a perspective, the microdirectional model describes the granular assembly as a collection of individual contacts oriented along various 
directions of the physical space. Further research showed that leaping from contact level directly to macroscopic scale involves loss of information about local proximity of particles. As such, reducing the microstructure of granular materials to a distribution of contact orientations does not sufficiently account for their complex mechanical behavior. With the development of numerical simulations based on discrete element methods (DEM), it was established that mesostructures composed of a few grains could be used to satisfactorily explain the macroscopic behavior of granular materials. Among such mesostructures, one can cite force chains (Peters et al., 2005; Tordesillas, 2007; Tordesillas and Muthuswamy, 2009; Wautier et al., 2017; Liu et al., 2020) or grain loops in 2D (Zhu et al., 2016a, 2016b; Liu et al., 2018). Moreover, recent studies (Pouragha and Wan, 2016, 2017, 2018a; Pouragha et al., 2019) demonstrate that the evolution of contact network due to various dissipative and non-dissipative mechanisms introduces new sources of non-linearity that can only be considered within modelling frameworks that transcends the conventional elasto-plastic theories (Pouragha and Wan, 2018b). Thus, for granular media with rapidly changing internal structure, an accurate understanding of the global constitutive behavior inevitably requires the inclusion of mesoscale interactions and their evolution at the intervening (meso) levels between single contact and macroscale.

Along this vein, the H-model (Nicot and Darve, 2011b) offers a simple, yet effective approach to enrich the initial microdirectional modelling framework with mesoscale physics. The model was initially developed in 2D to describe dry cohesionless granular materials as a collection of hexagonal mesostructures of six grains oriented along different directions in the physical space. Such mesostructures can be regarded as two force chains forming a grain loop, which makes the model consistent with DEM findings. The model has been recently extended to $3 \mathrm{D}$ with mesostructures of ten grains consisting of two imbricated hexagons in perpendicular directions (Xiong et al., 2018). Once implemented as suitable computational framework, such as FEM or Finite Difference based (FLAC), the model can be readily adopted to simulate a variety of geotechnical problems where multi-physical couplings can occur. Moreover, one main advantage of such approaches is to give access to a rich microstructural information at some given points of interest when dealing with a non-homogeneous boundary value problem. In essence, this information is out of reach for standard phenomenological models in which the microstructure is simplistically embedded as internal variables, and is not described in an explicit way. After a brief review of the standard 2D H-model, including comments of the recent 3D and multi-physics extensions, the manuscript shows how this approach is relevant to address 
boundary value problems in Geotechnics, and can effectively contribute to a better design of geotechnical structures.

\section{Multiscale framework: H-model}

\subsection{Model concept}

Different from phenomenological approaches that operate directly at the material point scale, multi-scale approaches formulate the constitutive relation of a material by averaging its behavior at a much smaller scale as illustrated in Figure 1. Such an upscaling process is known as homogenization. It supposes that the local behavior as well as the microstructure geometry of the considered material are already known. Thus, the derivation of the macroscale constitutive behavior requires the detailed description of the material's microstructure at the scale of a representative elementary volume (REV).

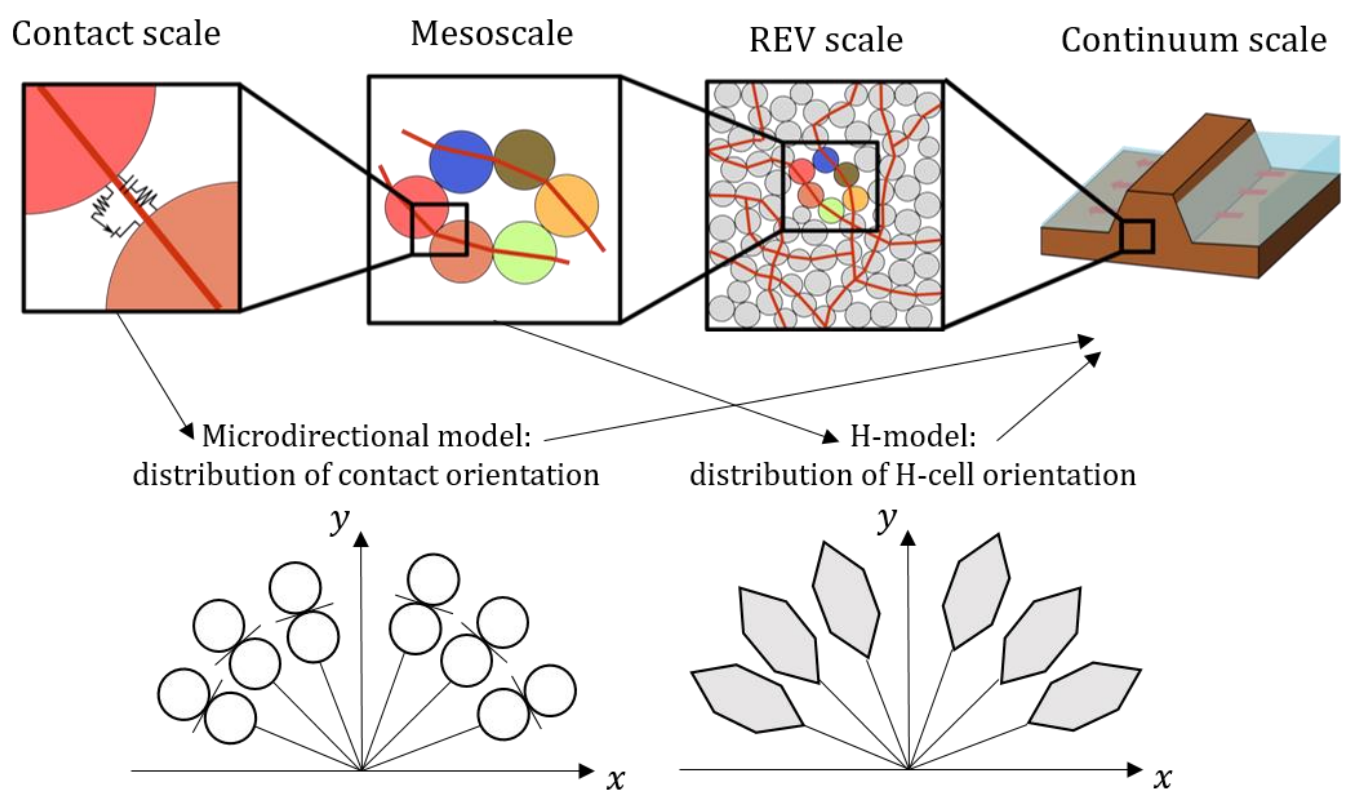

Figure 1. Hierarchical scales in granular materials. Phenomenological approaches deal with the continuum scale directly, spatial homogenization with the REV scale and statistical homogenization with the contact scale (microdirectional model (Nicot and Darve, 2005)) or mesoscale (H-model (Nicot and Darve, 2011b)).

Usually, homogenization is performed by spatial (volume) averaging over a representative elementary volume (REV). For instance, a cubical volume element of granular material can be modelled following a DEM approach as a collection of thousands of solid particles interacting 
through contact laws. Then, the macroscopic constitutive behavior is derived by computing the mean stress and strain values over the cubical domain.

The general idea behind the $\mathrm{H}$-model is to replace the above spatial description of a granular material with a statistical view at the mesoscale. Instead of explicitly considering all the particles in the entire granular assembly, H-model proposes to model granular media as a collection of independent mesostructure units composed of a few grains and oriented in different directions, as illustrated in Figure 1. The homogenized behavior of the collection of $\mathrm{H}$-cells is then obtained by directional averaging, and not spatial averaging as it is usually done in DEM simulations.

For the 2D H-model, the unit mesostructure is a hexagonal cell composed of six grains interacting through elasto-frictional contacts and kept at mechanical equilibrium with ten external forces as illustrated in the following Figure 2.
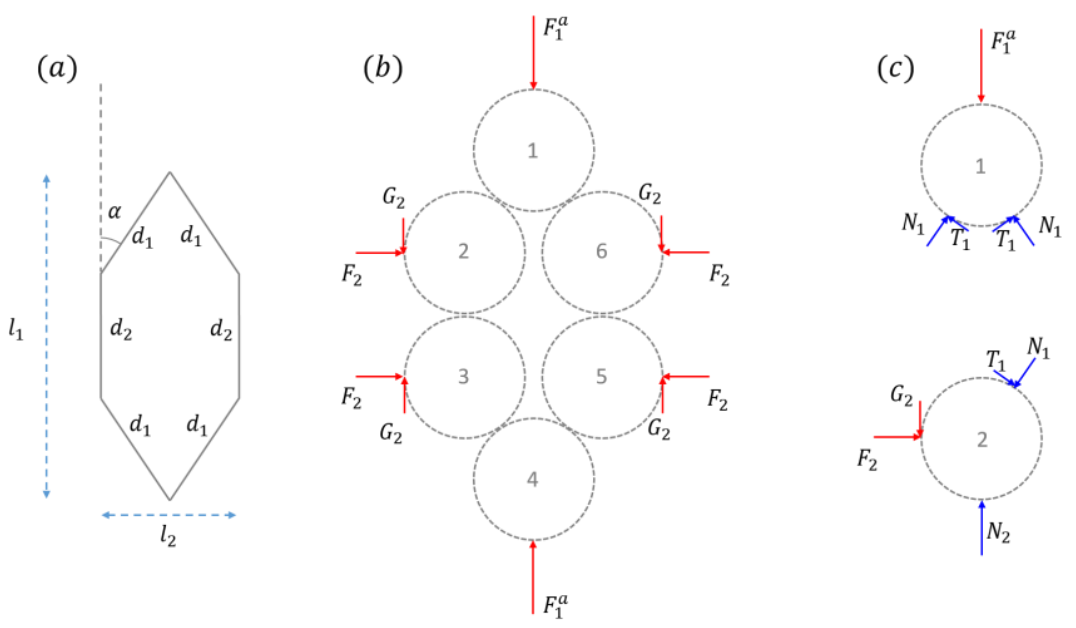

Figure 2. Hexagonal mesostructure as an assembly of six rigid disks in the H-model.

External (red) and internal (blue) forces necessary to keep the H-cell at equilibrium.

The choice to use mesostructures (and not simply independent contacts as done in the previous micro-directional model developed by Nicot and Darve (2005)) stems from the fact that the deformation of granular systems involves geometrical effects produced by the collective displacement of grains, which is consistent with strain definition in continuum mechanics. The particular choice of a hexagonal cell is based on the role played by grain loops of more than six grains as highlighted in recent micromechanical studies (Zhu et al., 2016a, 2016b; Liu et al., (2018)). Furthermore, this special mesostructured arrangement allows for an analytical 
description of the cell behavior, as recalled in the Appendix. The H-model was first introduced by Nicot and Darve (2011b) in 2D, then extended to 3D by Xiong et al. (2018). Research on the H-model is ongoing with recent developments aimed at enriching the microscale physics to include the effects of a fluid phase such as capillary phenomenon (Xiong et al., 2020). Preliminary results have also been obtained to account for internal erosion processes.

The general scheme of the standard 2D H-model is summarized in Figure 3 and reviewed in detail in the Appendix. The main features are listed as follows.
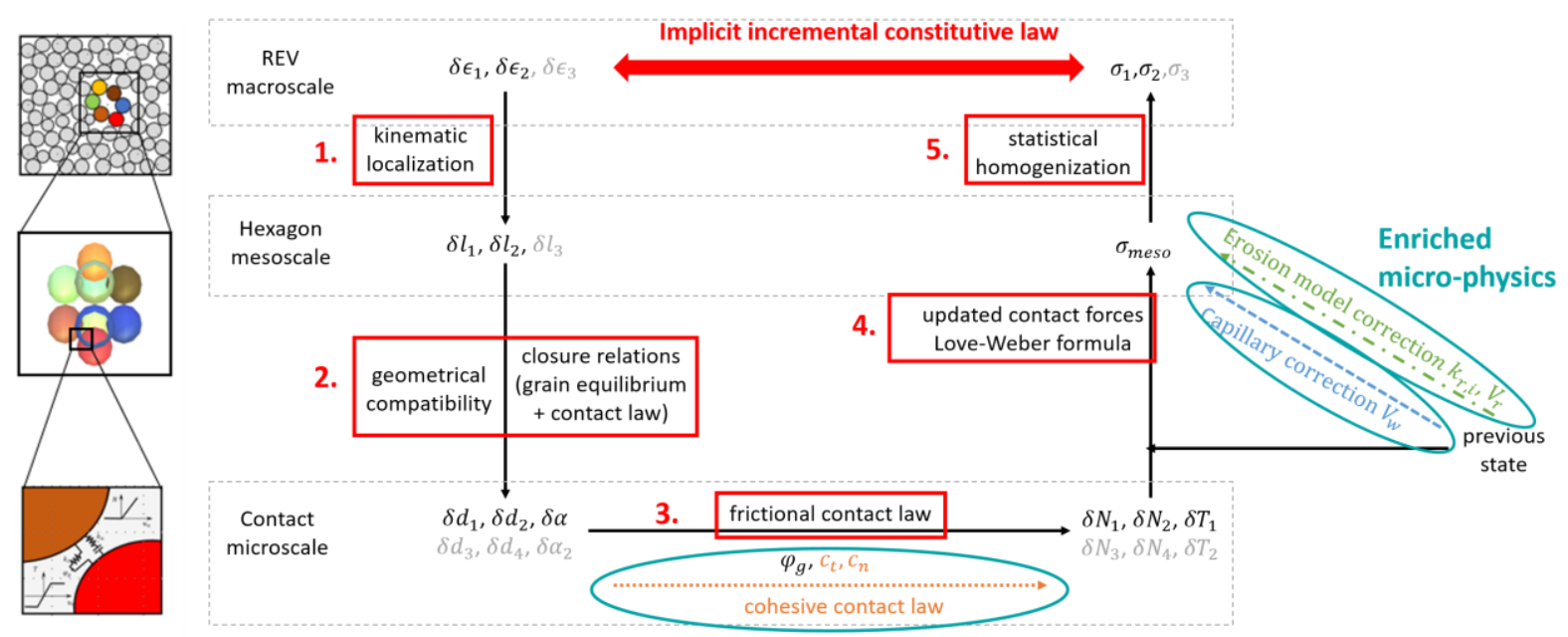

Figure 3. Five steps homogenization scheme of the H-model. Additional variables for the three-dimensional case are shown in grey. Current developments on the enriched versions of the model are highlighted.

1. The kinematic localization hypothesis consists of updating the H-cell dimensions of all the hexagons based on the macroscopic strain increment.

2. The resulting relative displacement of the grains of each H-cell are then computed by enforcing two geometrical compatibility equations and one closure relation ensuring the static equilibrium of the six grains.

3. Based on the incremental evolution of the H-cell geometry, the normal and tangential contact forces are updated.

4. A mesostress is then defined based on the application of Love-Weber formula for each H-cell 
5. The macroscopic stress is eventually obtained by statistical averaging of all the mesostresses. In this step, a weight function $\omega(\theta)$ depending on the orientation $\theta$ of the $\mathrm{H}$-cells is used to account for potential anisotropy of the microstructure.

\subsection{Model parameters}

The 2D H-model relies on three contact scale parameters $\left(k_{n}, k_{t}\right.$, and $\left.\varphi_{g}\right)$ and on the initial opening angle $\alpha^{\text {init }}(\theta)$ (geometric parameter) of each H-cell. Additionally, the probability density function $\omega(\theta)$ describing the statistical distribution of unit H-cells is needed. Inspired by contact scale analyses (Oda et al., 1985; Bathurst and Rothenburg, 1992), the contact distribution is usually approximated with a second order harmonic expansion. As a result, it is assumed that the initial distribution of unit H-cells can be written in a similar form, i.e.

$$
\omega(\theta)=\frac{1}{\pi}\left[1+a_{\omega} \cos 2\left(\theta-\beta_{\omega}\right)\right]
$$

where $a_{\omega}$ is a parameter ranging from 0 to 1 describing anisotropy of the hexagonal cell distribution, and $\beta_{\omega}$ its major principal direction. In practice, the distributions are discretized in $n_{\theta}$ directions $\theta_{i}$.

Note that Equation (1) does not exactly correspond to the contact distribution within a collection of unit H-cells. The contact distribution is rather a function of the probability density of the hexagonal cells described by $\omega(\theta)$ and the statistics of opening angles $\alpha(\theta) . \omega(\theta)$ is set once for all hexagonal units, while $\alpha(\theta)$ evolves with strain loading history through according to kinematical constraints (see Equations (A.6) and (A.7)).

The initial value of the opening angle should be related to porosity. Recalling that the mesovolume is assumed to be the bounding box of the inner hexagonal loop going through all grain centers, each of the six grains is half included in $V_{\text {meso }}$ if $0<\alpha<\pi / 3$ and $V_{s}=6 V_{g} / 2$. However, for $\pi / 3<\alpha<\pi / 2$ the lateral grains contribute less, i.e. only $1 / 4$ of their volume. Assuming the particles to be cylinders of diameter $D$ and unit height, the porosity reads

$$
\phi_{\text {meso }}=\frac{V_{\text {meso }}-V_{s}}{V_{\text {meso }}}=1-\frac{3 \pi D^{2}}{4 l_{1} l_{2}}, \quad \text { for } \alpha<\frac{\pi}{3}
$$

which leads in the initial state where $d_{1}=d_{2}=D$ to

$$
\phi_{\text {meso }}^{\text {init }}=1-\frac{3 \pi}{8 \sin \alpha^{\text {init }}\left(1+2 \cos \alpha^{\text {init }}\right)}, \quad \text { for } \alpha<\frac{\pi}{3}
$$

For $\pi / 3<\alpha<\pi / 2$, the analytical expression is more complicated, i.e.

$$
\phi_{\text {meso }}^{\text {init }}=1-\frac{3 \pi-(\beta-\sin \beta)}{8 \sin \alpha^{\text {init }}\left(1+2 \cos \alpha^{\text {init }}\right)}, \quad \text { for } \alpha>\frac{\pi}{3}
$$


where $\beta=2 \cos ^{-1}\left(2 \cos \alpha^{\text {init }}\right)$.

Combined with the probability density function $\omega(\theta)$, the macroscopic porosity $\phi$ is thus obtained as

$$
\phi=\int_{0}^{\pi} \omega(\theta) \phi_{\text {meso }} \mathrm{d} \theta
$$

The macroscopic void ratio is readily obtained as $e=\phi /(1-\phi)$.

Note that, at the scale of the H-cell, the expression of the local porosity $\phi_{\text {meso }}$ depends on the definition of $V_{\text {meso }}$. Consequently, the definition of $V_{\text {meso }}$ affects the macroscale interpretation of the model parameters $\alpha^{\text {init }}(\theta)$ and $\omega(\theta)$.

\section{H-model implementation and calibration}

The H-model was implemented in FLAC, a finite difference method (FDM) based program, to solve boundary value engineering problems. The FDM formulates the problem at hand in the dynamic regime so that an explicit scheme can be adopted to solve the equations of motion at each time step. At a given time step, the nodal displacements in a mesh are interpolated to define a piecewise constant strain field. For each element, the stress tensor is then estimated based on the material constitutive behavior. This is where the H-model is readily implemented following the scheme recalled in Figure 3 replacing classical phenomenological constitutive laws. From the piecewise constant stress field thus defined, nodal forces are calculated (this is done automatically in FLAC by integrating the stress vector along the edges of the mesh). Eventually, the mesh node positions are updated by integrating Newton's equation over an additional time step. To address static problems, non-viscous damping forces $F^{d}$ are added at each integration point to avoid any oscillations. The $i$-th component of such forces reads $F_{i}^{d}=-\kappa F_{i}^{u n} v_{i} /\|v\|$ where $\kappa$ is a damping ratio, $F^{u n}$ the unbalanced force, and $v /\|v\|$ returns the direction of the velocity. A typical value $\kappa=0.8$ is used.

\subsection{Calibration using plane strain drained experiments}

Before examining the 2D H-model for engineering structures in plane strain conditions, the present section evaluates the model performance at the material point scale. A careful attention is paid here to assess the extent to which the $2 \mathrm{D}$ version of the H-model can quantitatively capture salient features of the mechanical response of real sands under plane strain conditions ${ }^{1}$.

\footnotetext{
${ }^{1}$ One of the intrinsic limitation of the model is that the out of plane stress component is not accounted for, as the stress tensor remains 2D by definition (see Equations (A.9) and (A.11)). 
The experiment was carried out on RF Hostun sand along plane strain drained compression loading paths (Desrues and Viggiani, 2004).

RF Hostun sand is a fine-grained, angular silica sand. The mean particle diameter $D_{50}$ is equal to $0.35 \mathrm{~mm}$ whereas the particle size distribution has a coefficient of uniformity $C_{u}=$ $D_{60} / D_{10}=1.70$ (Calvetti et al., 1997). The conventional minimum and maximum unit weights are 13.24 and $15.99 \mathrm{kN} / \mathrm{m}^{3}$, respectively, whereas the specific gravity $G_{S}$ is 2.65 . Therefore, the maximum and minimum void ratios range between $e_{\max }=0.963$ and $e_{\min }=0.626$. For later comparison, it is important to keep in mind that these void ratios are $3 \mathrm{D}$, whereas those derived from the H-model are 2D (for 2D/3D comparisons it is thus more relevant to consider the relative densities).

The tests on water-saturated RF Hostun sand were conducted at both dense and loose states with initial void ratio ranges of $e_{0} \in[0.631-0.644]$ and $e_{0} \in[0.850-0.865]$, respectively. The effective confining stress was $\sigma_{3}^{\prime} \in\{100,200,400\} \mathrm{kPa}$. The discussion of results makes use of the following stress invariants:

$$
\left\{\begin{array}{l}
s^{\prime}=\frac{\sigma^{\prime}{ }_{1}+\sigma_{3}^{\prime}}{2} \\
t=\frac{\sigma_{1}^{\prime}-\sigma_{3}^{\prime}}{2}
\end{array}\right.
$$

where $t$ and $s^{\prime}$ correspond to the so-called Roscoe's deviatoric stress and mean effective stress, respectively.

The calibration of the model was carried out using experimental results obtained at a confining pressure $\sigma_{3}^{\prime}=200 \mathrm{kPa}$. In the experiments, strain localization was observed with considerable softening, signaling shear band formation. As soon as a shear band forms, the sample does not behave homogeneously anymore. Therefore, the experimental stress-strain curves correspond to the response of a structure that should be modeled as such. Given that the $\mathrm{H}$-model is formulated at the material point scale, its calibration is carried out based only on the pre-localization part of the experimental curve.

The first step of the calibration refers to the elastic parameters, i.e. the material stiffness and compressibility in the small strain range. Then, the inter-granular friction angle is chosen to reproduce the peak value of the deviatoric stress. Eventually, the microstructural parameters are determined iteratively to calibrate the axial strain corresponding to the peak in the deviatoric stress. It goes without saying that the same contact law parameters are used for simulating both loose and dense samples. It should also be acknowledged that calibrating the volume response 
curve was not attempted, given that the H-model is a 2D model, the volumetric behavior of which may be significantly different from those of 3D materials.

Table 1 summarizes the parameters obtained from the calibration procedure. It can be seen that parameters describing the inter-granular contact law correspond to values broadly used in DEM simulations.

Table 1. Summary of the experimental program used for the model calibration and validation

\begin{tabular}{|c|c|c|c|}
\hline Parameter & Symbol & Loose & Dense \\
\hline Normal contact stiffness & $k_{n}$ & \multicolumn{2}{|c|}{$150 \mathrm{MN} / \mathrm{m}$} \\
\hline Contact stiffness ratio & $k_{t} / k_{n}$ & \multicolumn{2}{|c|}{0.5} \\
\hline Inter-granular friction angle & $\tan \varphi_{g}$ & \multicolumn{2}{|c|}{0.5} \\
\hline Anisotropy of the distribution & $a_{\omega}$ & 0.5 & 1.0 \\
\hline Principal direction of anisotropy & $\beta_{\omega}$ & $90^{\circ}$ (hor.) & $0^{\circ}$ (vert.) \\
\hline Initial opening angle & $\alpha_{0}$ & $60.0^{\circ}$ & $35.0^{\circ}$ \\
\hline
\end{tabular}

Referring to Table 1, one should notice that in the loose case, the initial opening angle is set to a large value representative of the maximum void ratio (see equations (3) and (4)). The hexagon distribution is slightly anisotropic and oriented horizontally. In the dense case, the initial opening angle is set close to the minimum opening angle $\left(\alpha=30^{\circ}\right)$ while the hexagonal unit cell distribution is much more anisotropic and oriented vertically as one could have expected for densely compacted specimens (vertical compaction direction in experimental procedure). The initial contact distributions for the dense and loose samples are shown in Figure 4. These values have been deduced from information on the hexagon distribution function $\omega(\theta)$ and the opening angle $\alpha(\theta)$ of the different H-cells.

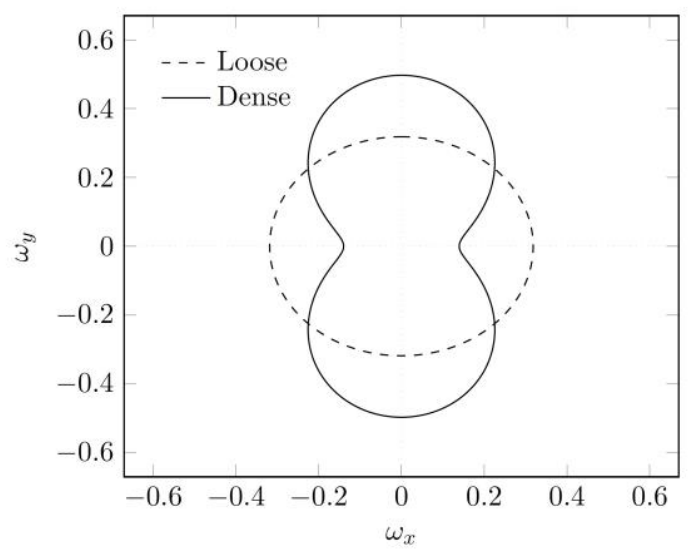

Figure 4. Initial contact distributions for the dense and loose sample parameters of Table 1. 


\subsection{Evaluation of the model performance}

To evaluate the predictive ability of the H-model, experimental curves at confining pressures $\left(\sigma_{3}^{\prime}=100\right.$ and $400 \mathrm{kPa}$ ) were considered while the test with ${\sigma^{\prime}}_{3}=200 \mathrm{kPa}$ has been used for calibration purposes. Figure 5 and Figure 6 show comparisons of numerical simulations and

In the loose case, the H-model calculations capture the general trend of the experimental stress-strain curves characterized by ultimate values of deviatoric stress. The H-model fails to reproduce the volumetric response as it underestimates the material's contractancy. However, the influence of the confining pressure on the contractancy is well captured.

For both dense and loose cases, we see that the model tends to overestimate the influence of the confining pressure on the small strain stiffness of the material. This suggests a nonlinear 
dependency between the normal reaction and the interpenetration of the grains. This feature could constitute a possible improvement of the H-model.
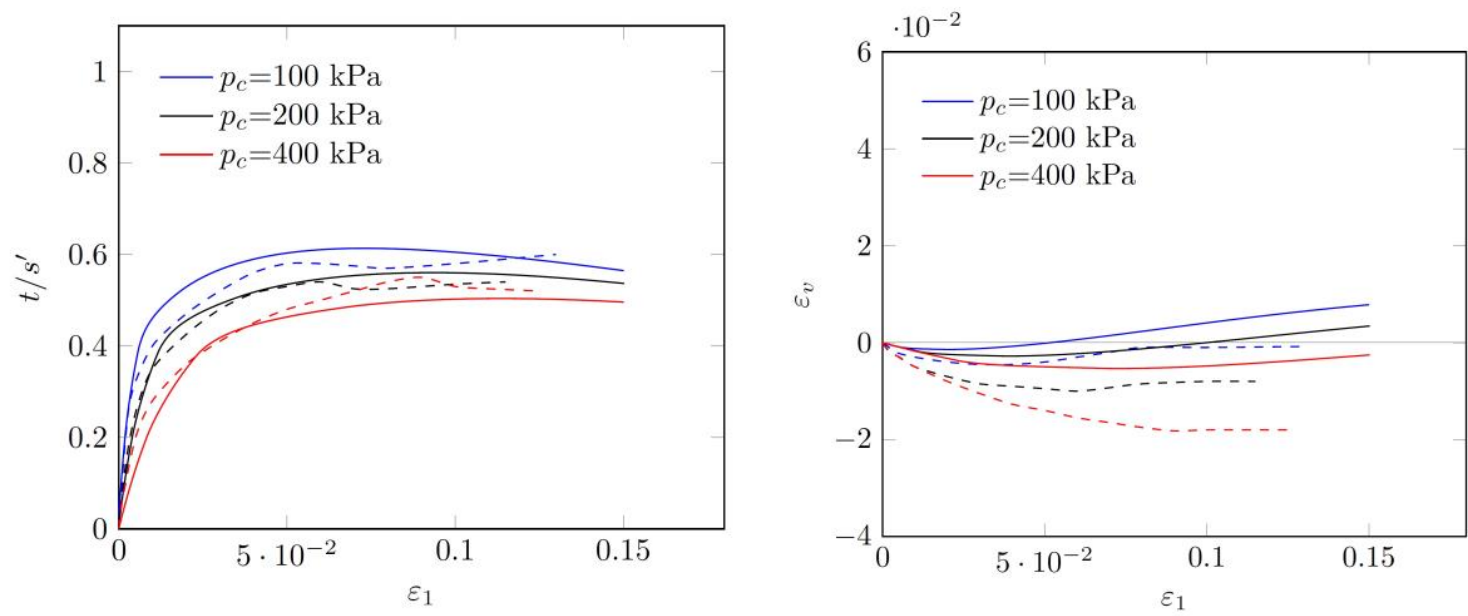

Figure 6. Comparison between experimental drained plane strain biaxial compression tests

(Desrues and Viggiani, 2004) and H-model simulations on loose Hostun RF sand. The experimental curves are in dashed lines and the H-model curves in solid lines. Calibration is done for $200 \mathrm{kPa}$ while predictions are shown for 100 and $400 \mathrm{kPa}$.

We also note that the H-model is unable to reproduce critical state, characterized by constant shear strength and constant volumetric strain at large deformations. This is an intrinsic limitation of the H-model at the material point scale. Indeed, critical state is a steady state from a macroscale point of view, which arises from permanent microstructure transformations at the lower scales. The microstructure reaches equilibrium only from a statistical point of view while mesostructures are constantly being rearranged. Improvements of the H-model are currently being worked out to find a rational way to embed microstructure transformations in the model.

The above-mentioned intrinsic limitation of the H-model, however, does not prevent its implementation and use in boundary value problems, even at large strain levels. At the structural scale, geometric effects arise in addition to material properties and could even become prominent. We therefore explored the predictive capacities of the calibrated H-model by considering the drained biaxial test as a boundary value problem using a non-homogeneous mesh domain. It is well known that the mesh size has an influence on the post-peak behavior of the modeled material. As a result, we have chosen the dimension corresponding to the width of the shear band, i.e. typically $10 D_{50}$ (see Alshibli et al. (1999) for instance). This condition corresponds to a mesh of $30 \times 100$ elements. At the beginning of the simulation, the material 
properties are homogeneous all over the mesh. The resulting stress and volumetric strain curves are shown in Figure 7 for one of the tests of Desrues and Viggiani (2004). Note that the material parameters used are those of Table 1 for all the mesh elements.
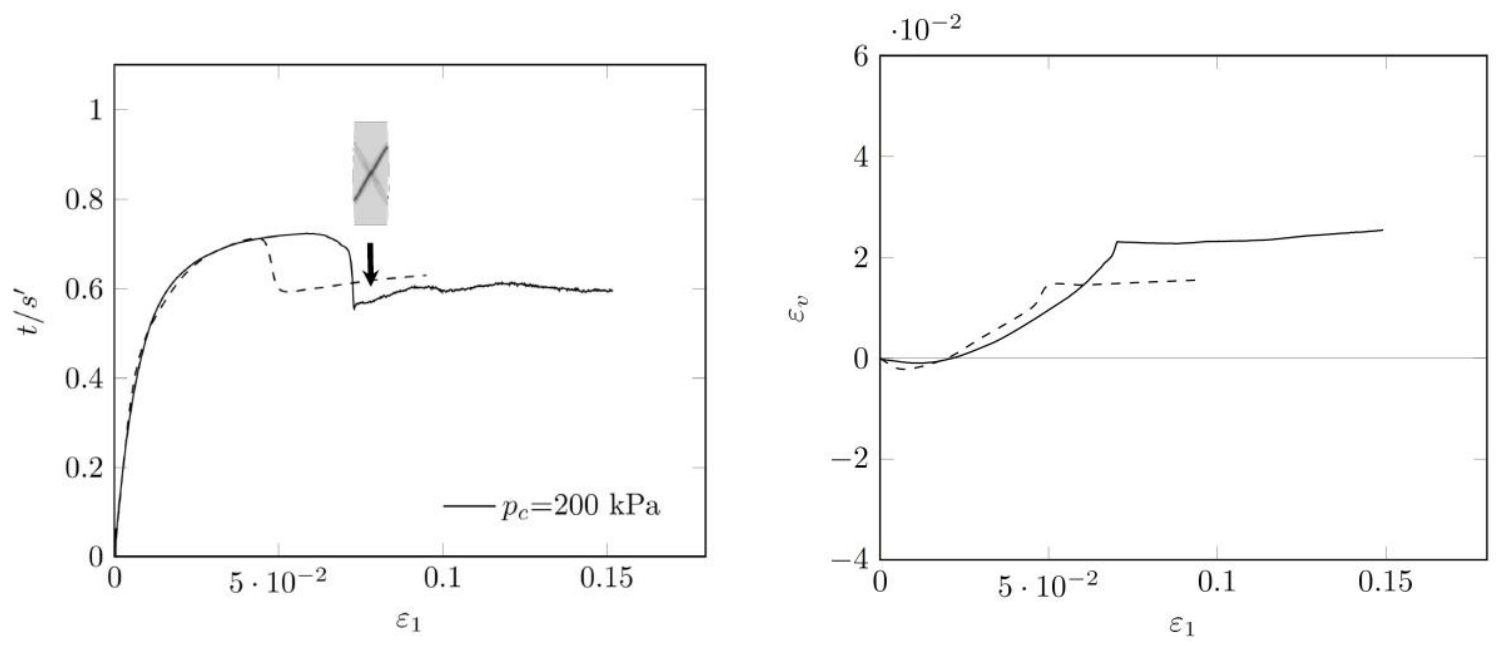

Figure 7. Drained plane strain biaxial compression for $\boldsymbol{\sigma}^{\prime}{ }_{3}=\mathbf{2 0 0} \mathbf{~ k P a}$ (Desrues and Viggiani, 2004) on dense Hostun RF sand. Comparison between experimental curve (dashed line) and numerical simulation for a 30 x 100 mesh (solid line).

We can see that the pre-peak behavior is preserved (this test was used for calibration at the material scale). In particular, the volumetric strain curve predicted by the H-model tends to underestimate the dilatancy of the material. However, we note that the added structural dimension to the modeling makes it possible to account for a steady state beyond an axial strain of $\varepsilon_{1} \approx 7 \%$, close to the $5 \%$ observed experimentally.

The emergence of a steady state in a boundary value setting demonstrates that the apparent softening observed experimentally is mostly linked to a transition from homogeneous states to strain localization. In this sense, experimental softening has more of a structural origin in the dense case than a material one (Sterpi, 1999). However, the material source of the softening cannot be disregarded since a small, yet critical material softening is embedded in the H-model (as seen in Figure 5 and Figure 6).

The structural response relies on the existence of minute numerical fluctuations in the local material properties, which act as a catalyzer for early strain localization and the associated deviatoric stress reduction. The prevalence of structural softening thus justifies the use of the $\mathrm{H}$-model, even at large strain levels. It also justifies the priority that was given to improve the 
H-model by extending it to 3D (Xiong et al., 2017) to better account for volume variations. These are relevant in the case of hydro-mechanical coupling, where volume variations control the pore water pressure under undrained conditions.

Overall, the present analyses demonstrate that the H-model can reproduce the mechanical response of a sand as long as it is used within the framework associated with the hypothesis of homogeneity of the small strain field. The extension to non-homogeneous cases can be achieved by adopting numerical simulations with an appropriate mesh. The appropriate dimension of the mesh should be small enough to simulate the failure modes expected (localization of the strains for example) while being limited by the internal length of the material. In the present case, we may define an upper limit of the mesh size equal to $1 / 10^{\text {th }}$ of the smallest dimension of the sample and a lower limit equal to $10 D_{50}$ in order to guarantee the representativeness of the elementary volume. In the present case, the minimal mesh size of $10 D_{50}$ was considered.

\subsection{Micromechanical inspection of the structure response}

One of the interesting features of the H-model is that it embeds meso-structure distributions in its formulation. As a result, some micromechanical statistical information can be readily extracted for each material point in structure scale computations ${ }^{2}$. For instance, while simulating the biaxial response of the dense sample and the shear band localization process in Figure 7, it is meaningful to analyze the mechanical state of the different H-cells inside and outside of the shear band. As seen in Erreur ! Source du renvoi introuvable., the proportion of H-cells in elastic, plastic or tensile deformation regimes are given in all the elements of the finite difference computation. Complementary to Erreur ! Source du renvoi introuvable., Erreur ! Source du renvoi introuvable. shows for two material points the different regimes corresponding to the directions of the H-cells.

\footnotetext{
${ }^{2}$ Without paying the large computation costs of DEM simulations. -15 -
} 


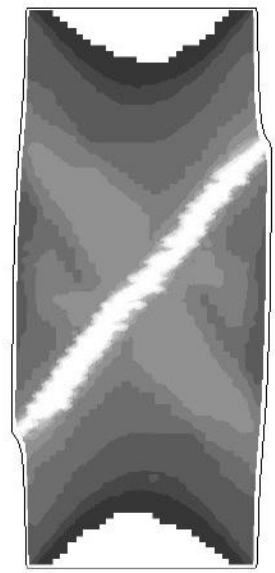

(a)

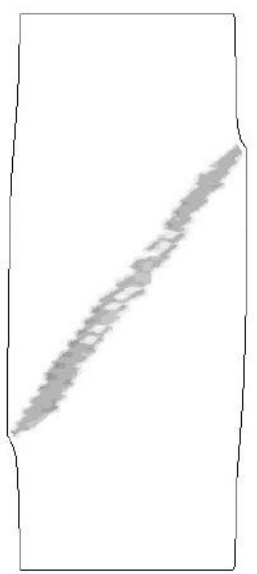

(b)

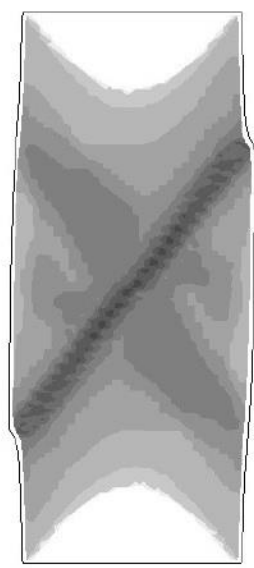

(c)

Figure 8. Proportion of hexagons in elastic (a), plastic (b) and tensile (c) regimes after the localization of deviatoric strains $\left(\varepsilon_{1}=0.08\right)$.
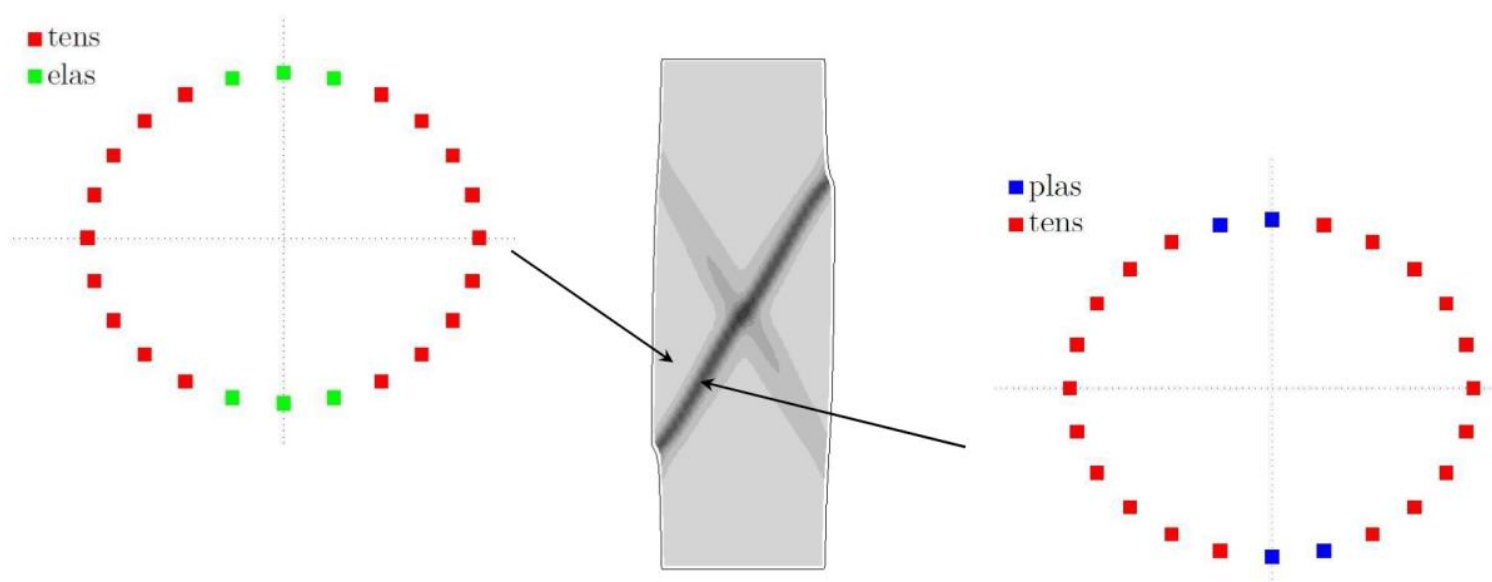

Figure 9. Directional analysis of the contact regimes of hexagons outside (left) and inside (inside) the shear band $\left(\varepsilon_{1}=0.08\right)$.

Figure 8 shows that most of the H-cells in which plasticity has been activated concentrate within the shear band domain while most of the H-cells located outside of the shear band domain remain within the elastic regime. In addition, the shear band domain contains a large proportion of H-cells in tension (in which contact loss has occurred). All these results are very consistent with DEM observations indicating that grain sliding and contact loss concentrates within the shear band (see for instance Liu et al., 2018 and Liu et al., 2020). Within the elastic 
zone, a significant proportion of $\mathrm{H}$-cells are in tension, which relates to the lateral expansion of the sample observed in drained biaxial loading.

Figure 9 indicates that contact loss mostly occurs for H-cells oriented along the horizontal direction and thus compressed along their local $\boldsymbol{t}$ direction (see Figure 2). As a result, contacts with grains 1 and 4 (see Figure 2) are lost, which corresponds to relatively horizontal contacts in the boundary value problem. Here again this observation is consistent with DEM results, showing that biaxial loading conditions induce horizontal contacts loss from the very beginning of the loading. This explains why contact loss also occurs in the elastic zone (Pouragha and Wan, 2017). In Figure 9, it should also be noted that the onset of the shear band breaks the symmetry for the regime distribution of the H-cells. This is linked to a local rotation of the principal stress and strain directions (observed through force chain rotation in Liu et al. (2020) for instance).

As for the previous micromechanical observations, it should however be noted that only a limited number of directions does not undergo tension in Figure 9. This is also in relation to the aforementioned limitation of the $\mathrm{H}$-model to account for microstructure dynamic reorganizations. However, the results presented in this section demonstrate that the H-model can be considered as a viable tool in the numerical modeling of engineering problems.

\section{Simulation of an engineering problem}

The purpose of this section is to illustrate the applicability of the H-model to engineering problems by considering the static liquefaction of an earth dam. A hydro-mechanical coupled simulation is performed using the FLAC software in which the soil constitutive behavior follows the H-model. The formulation of coupled fluid-mechanical processes is done within the framework of the quasi-static Biot's theory. The fluid phase is defined by a volumetric weight, a bulk modulus and tension limit. The hydraulic properties of the solid phase is characterized by a Darcy permeability, irrespective of the microstructural variables used in the $\mathrm{H}$ model. No spatial heterogeneities are considered in the hydraulic properties of the material.

\subsection{Model set up}

A homogeneous dam built on a two layered foundation is considered. The dam body and the foundation geometry are described as shown in Figure 10, while the corresponding numerical values are summarized in Table 2. 


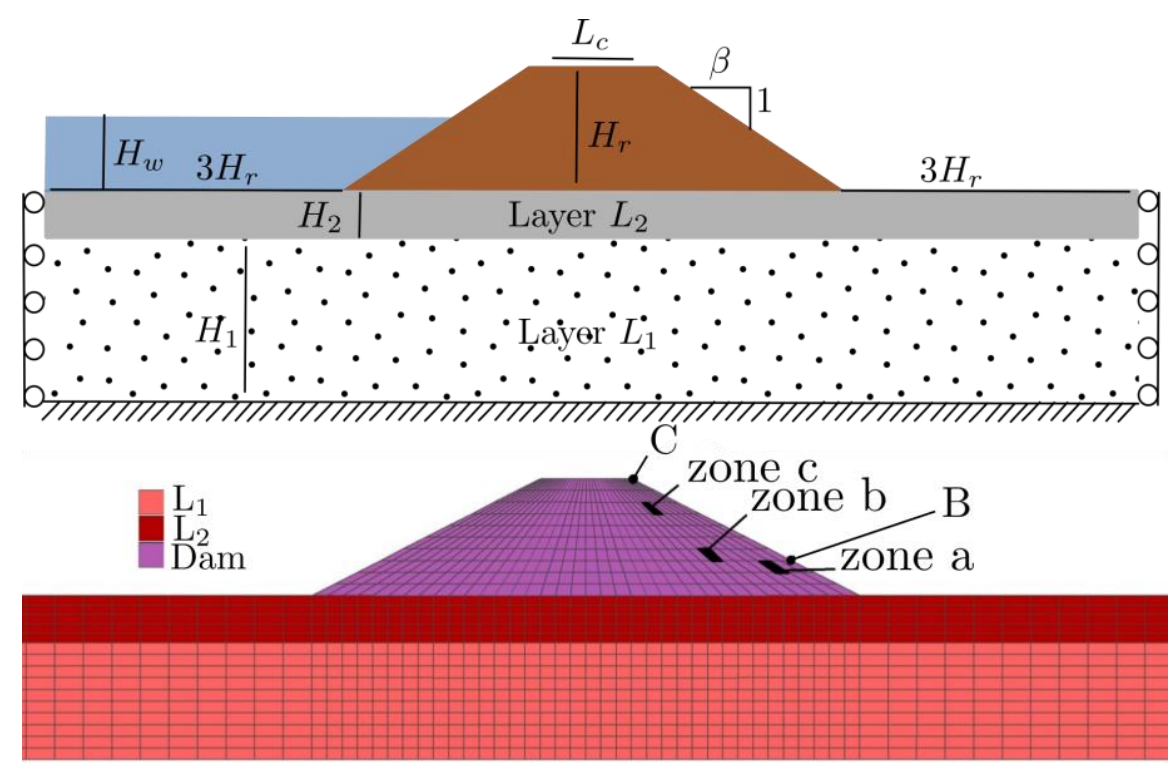

Figure 10. Geometric view of the dam, reservoir and foundation, and the corresponding mesh.

Table 2. Geometric parameters of the dam and its foundation.

\begin{tabular}{lcc}
\hline Parameter & Symbol & Value \\
\hline Dam height & $H_{r}$ & $5 \mathrm{~m}$ \\
Crest width & $L_{c}$ & $4 \mathrm{~m}$ \\
Slope & $\beta$ & 2.5 \\
Layer 1 thickness & $H_{1}$ & $5 \mathrm{~m}$ \\
Layer 2 thickness & $H_{2}$ & $2 \mathrm{~m}$ \\
Water height & $H_{w}$ & {$\left[0, H_{r}\right]$} \\
\hline
\end{tabular}

The foundation soil consists of two layers that are assumed to follow a standard elasto-plastic Mohr-Coulomb material while the dam body behaves according to the H-model. The material parameters used in this section are summarized in Table 3.

Table 3. Material parameters for the two layers of the foundation and for the dam body.

\begin{tabular}{clccc}
\hline Material / type & \multicolumn{1}{c}{ Parameter } & Symbol & Unit & Value $\left(L_{1}\right.$ or $\left.L_{2}\right)$ \\
\hline & Dry density & $\rho_{d}$ & $\mathrm{~kg} / \mathrm{m}^{3}$ & 1700 \\
& Compressibility modulus & $K$ & $\mathrm{MPa}$ & 200 \\
Layer $L_{1}$ or Layer $L_{2}$ & Shear modulus & $G$ & $\mathrm{MPa}$ & 100 \\
Mohr-Coulomb & Effective cohesion & $c^{\prime}$ & $\mathrm{kPa}$ & 0 \\
& Friction angle & $\varphi$ & Degrees & 35 or 30 \\
& Dilatancy angle & $\psi$ & Degrees & 5 or 0
\end{tabular}




\begin{tabular}{clccc} 
& Permeability & $k$ & $\mathrm{~m} / \mathrm{s}$ & $10^{-6}$ or $10^{-7}$ \\
\hline \multirow{3}{*}{ Dam body } & Dry density & $\rho_{d}$ & $\mathrm{~kg} / \mathrm{m}^{3}$ & 1600 \\
H-model & Normal stiffness & $k_{n}$ & $\mathrm{MN} / \mathrm{m}$ & 100 \\
& Tangential stiffness & $k_{t}$ & $\mathrm{MN} / \mathrm{m}$ & 50 \\
& Contact friction angle & $\varphi_{g}$ & Degrees & 30 \\
& Initial opening angle & $\alpha^{\text {init }}$ & Degrees & 50 \\
& Anisotropy coefficient & $a_{\omega}$ & - & 0 \\
& Principal direction of anisotropy & $\beta_{\omega}$ & Degrees & 0 \\
& Permeability & $k$ & $\mathrm{~m} / \mathrm{s}$ & $10^{-6}$ \\
\hline
\end{tabular}

The discretization of the dam and foundation comprises 1260 rectangular elements and 1341 nodes (see Figure 10). The bottom of layer $L_{1}$ is fixed while the lateral boundaries of $L_{1}$ and $L_{2}$ are only constrained in the horizontal plane, but free to dilate in the vertical direction. In order to avoid any influence of the boundary conditions on the dam, the lateral extents of the problem are set to $3 H_{r}$ and the thickness of $L_{1}$ is set to $H_{1}=H_{r}$. For hydraulic part, impermeable boundaries are assigned to the bottom and lateral sides of the foundation. A zero water pressure value is applied on the downstream side of the dam and the foundation, while a hydrostatic water pressure is applied in the water reservoir.

\subsection{Simulation procedure}

Following an explicit numerical scheme, the dynamic equations of motion are solved at each time step. As a result, the numerical computation of the static equilibrium of the dam is based on a three-step procedure as follows, illustrated in Figure 11.

1. The stress field in the dry foundation is initialized before the dam is built. This is done by turning on gravity such that the initial stress field satisfies a given value of lateral earth pressure at rest. This loading step is completed once convergence to equilibrium is achieved.

2. The dam is built in lifts by activating gravity in successive layers of $0.50 \mathrm{~m}$, which mimics the actual construction of a dam. Here again, the computations will move to the next lift only when convergence to equilibrium is ensured.

3. The filling of the reservoir is modelled by raising the water level gradually. The hydromechanical computation is conducted in an uncoupled way, except for the last raising of the water height to $H_{w}=H_{r}$. First, the pore water pressure field is computed for a given dam geometry based on the material permeability $k$. Then, this pore water 
pressure field is used to compute equivalent nodal forces based on local pressure gradients that act on the mesh nodes. These forces are in turn taken into account as external nodal forces in the mechanical computation. Both the dam and foundation deform, and the stress field is updated after a new equilibrium configuration is reached. If the computations do not converge, the dam is then deemed to fail.

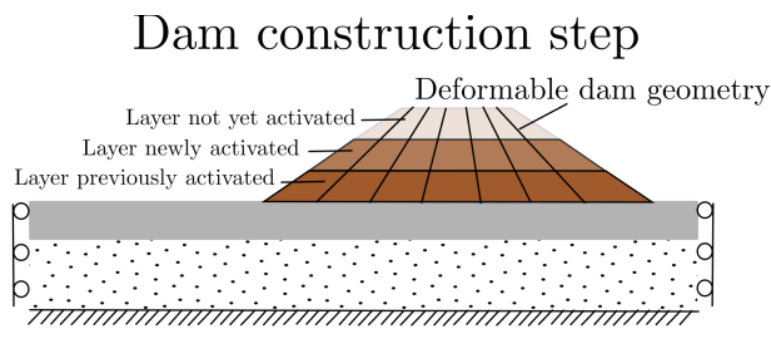

Reservoir filling step
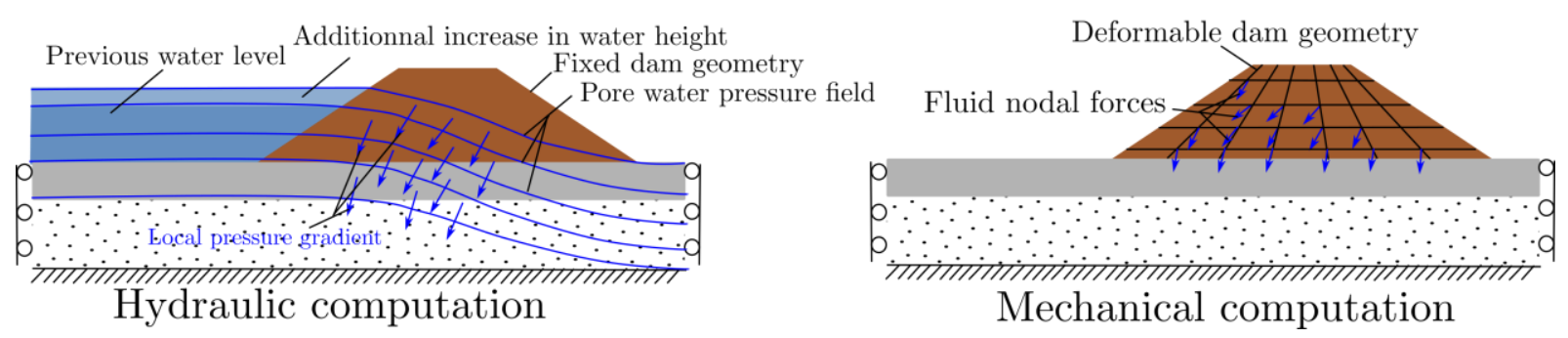

Figure 11. Simulation steps.

\subsection{Results}

Figure 12 shows the effective stress fields obtained at the end of the dam construction and after the water height reaches $H_{w}=4.5 \mathrm{~m}$. 


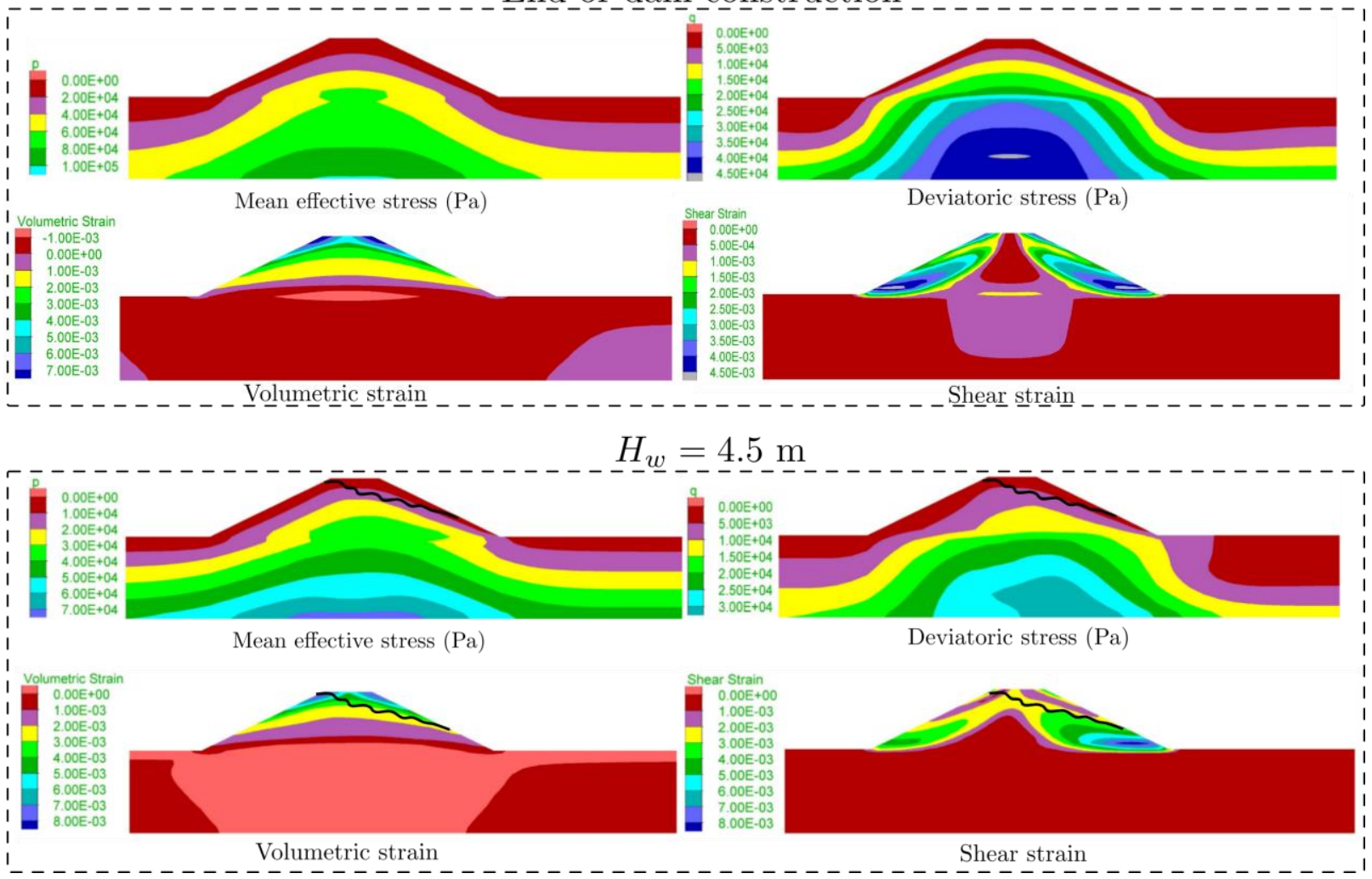

Figure 12. Stress and strain fields at: (a) end of dam construction (top), (b) filling of dam when the water height reaches $\boldsymbol{H}_{\boldsymbol{w}}=\mathbf{4 . 5} \mathbf{~ m}$ (bottom). The black solid line corresponds to the piezometric level.

As expected, all stress fields satisfy the symmetry condition after the dam construction (Figure 12). The maximum displacement at the bottom right and left of the dam reaches a few centimeters laterally, i.e. about $1.4 \mathrm{~cm}$ around point B (location shown in Figure 10). The dam material dilates just after being put in place, before contracting as it is buried under successive layers of material (maximum contraction of about $0.1 \%$ at the base of the dam as shown in Figure 12). Such results are consistent with actual observations and standard numerical simulations.

As the water level increases, the problem's symmetry is broken due to pore-water pressure difference between upstream and downstream domains. The pore water pressure increases more in the upstream than in the downstream part of the dam, which results in a decrease/increase in the effective stress in the upstream/downstream side of the dam respectively (Figure 12, bottom left). At equilibrium, the horizontal displacements reach a maximum of $2.4 \mathrm{~cm}$ around point $\mathrm{B}$ (location shown in Figure 10). In this situation, the fraction of H-cells in which sliding activates remains equal to zero percent but the fraction $f_{\text {open }}$ of H-cells that are under tension (contact 
opening) reaches $70 \%$ on the dam surface and decreases with depth. Such a fraction is expressed as $f_{\text {open }}=\int_{0}^{\pi} \omega(\theta) \delta_{\text {loss }}(\theta) \mathrm{d} \theta$ where the $\delta_{\text {loss }}$ function equals to 1 if the contact loss condition is met for the H-cell in direction $\theta\left(d_{1}(\theta)>D\right.$ or $\left.d_{2}(\theta)>D\right), 0$ otherwise. Figure 13 shows the evolution of this ratio during the filling of the reservoir.

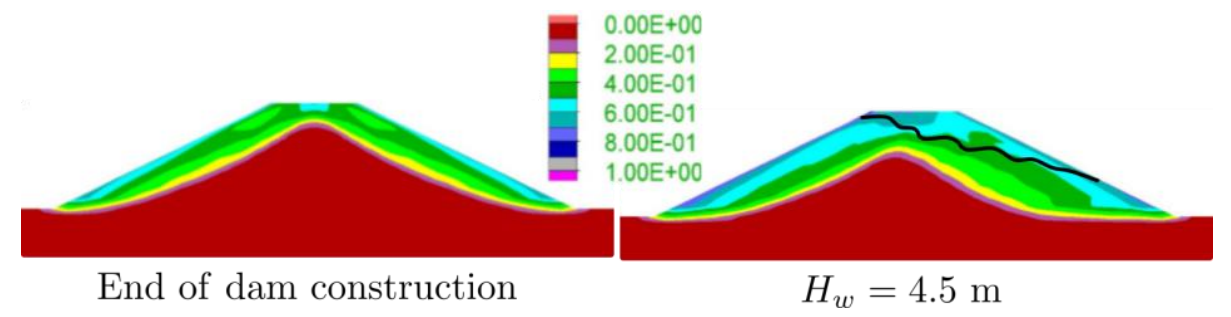

Figure 13. Proportions $\boldsymbol{f}_{\text {open }}$ of the H-cells in which contact has been lost after the end of the dam construction (left) and when the water height reaches $\boldsymbol{H}_{\boldsymbol{w}}=\mathbf{4 . 5} \mathbf{~ m}$ (right). The black solid line corresponds to the piezometric level.

For the last incremental elevation of the water level $\left(H_{w}=H_{r}\right)$, failure is observed. For this particular step, a fully coupled hydro-mechanical computation is performed, with an update of the pore water pressure field, at each time step. Figure 14 shows the time evolution of the displacements of points B and C (see Figure 10).

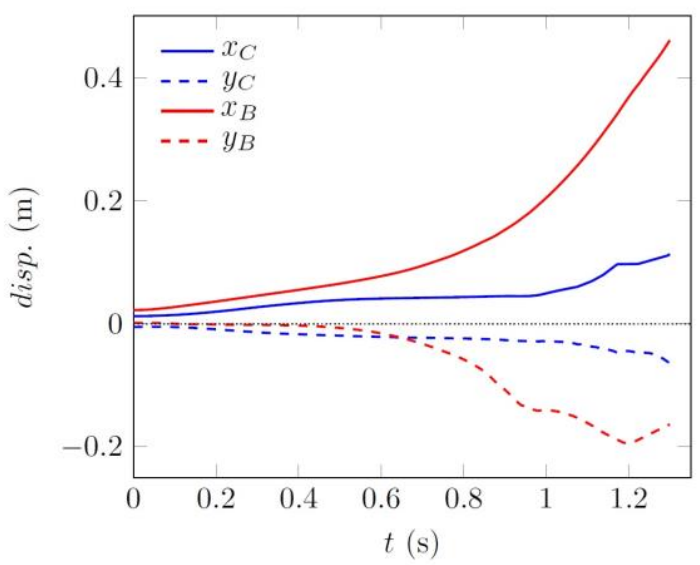

Figure 14. Time evolution of the displacement of points B and C (see Figure 10) when

$$
H_{w}=H_{r} .
$$

After only $1.3 \mathrm{~s}$, the displacement at point B reaches nearly $50 \mathrm{~cm}$. Figure 15 shows the stress, strain and displacement fields at this time, while the proportion of sliding $\left(f_{\text {slide }}\right)$ and opening $\left(f_{\text {open }}\right) \mathrm{H}$-cells at failure is given in 
Figure 16. Similar to $f_{\text {open }}$, the sliding proportion of $\mathrm{H}$-cells is expressed as $f_{\text {slide }}=$ $\int_{0}^{\pi} \omega(\theta) \delta_{\text {slide }}(\theta) \mathrm{d} \theta$ where the $\delta_{\text {slide }}$ function equals to 1 if the contact sliding condition is met for the H-cell in direction $\theta\left(\left|T_{1}(\theta)\right|=N_{1}(\theta) \tan \varphi_{g}\right), 0$ otherwise.

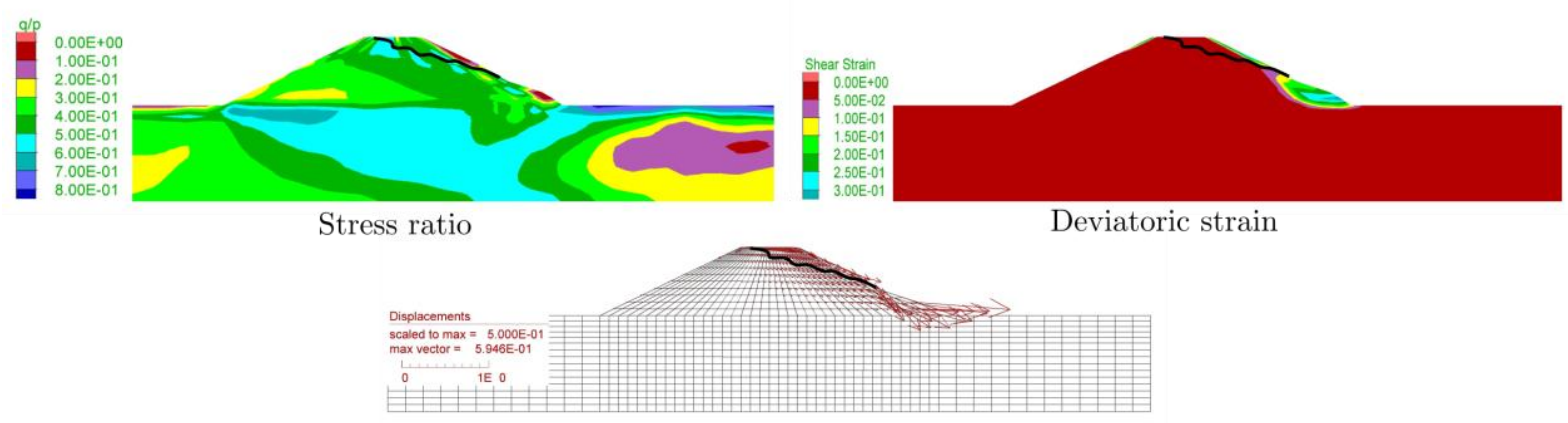

Figure 15. Stress ratio, deviatoric strain and displacement fields at failure $\left(\boldsymbol{H}_{\boldsymbol{w}}=\boldsymbol{H}_{\boldsymbol{r}}\right.$ and

$$
\boldsymbol{t}=1.3 \mathrm{~s}) \text {. }
$$

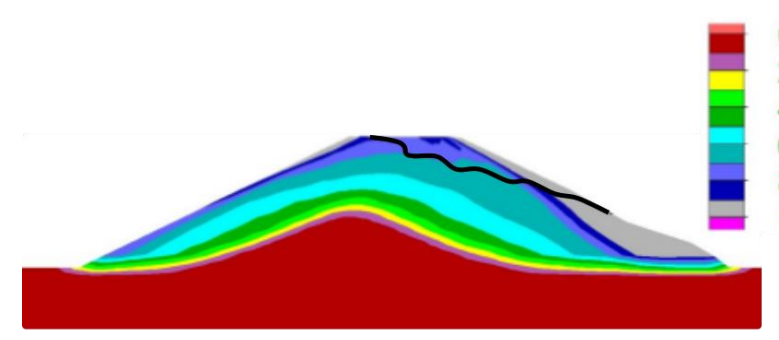

Opened $\mathrm{H}-$ cells

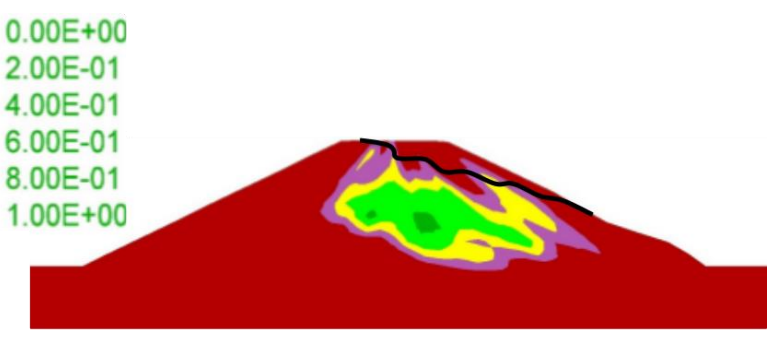

Sliding H - cells

Figure 16. Proportions of the H-cells in which contact has been lost $\left(\boldsymbol{f}_{\text {open }}\right.$, left) or sliding has occurred $\left(\boldsymbol{f}_{\text {slide }}\right.$, right $)$ at failure $\left(\boldsymbol{H}_{\boldsymbol{w}}=\boldsymbol{H}_{\boldsymbol{r}}\right.$ and $\left.\boldsymbol{t}=\mathbf{1 . 3 \mathrm { s }}\right)$. The black solid line corresponds to the piezometric level.

It can be noticed that failure on the downstream side of the dam does not correspond to a large proportion of sliding $\mathrm{H}$-cell, but to a very large proportion of opening H-cells (nearly 100 $\%$ on the downstream face of the dam). This shows that the failure mechanism does not correspond to sliding (when the plastic limit criterion is reached) but to a static liquefaction (massive loss of inter-granular contacts) which is confirmed in Figure 17 by looking at the loading path followed by the material located in the bottom right of the dam ("zone a" in Figure 10). The results demonstrate the ability of the H-model to capture this particular type of failure mechanism, which is often overlooked in standard mechanical stability analysis of such engineering structures. This also shows how the H-model can be used to account for some of 
the most relevant mesoscale features as well as to pinpoint the local scale failure mechanisms in a boundary value engineering problems at a cheap computational cost (much cheaper than DEMxFEM computations for instance ; Guo and Zhao, 2014).

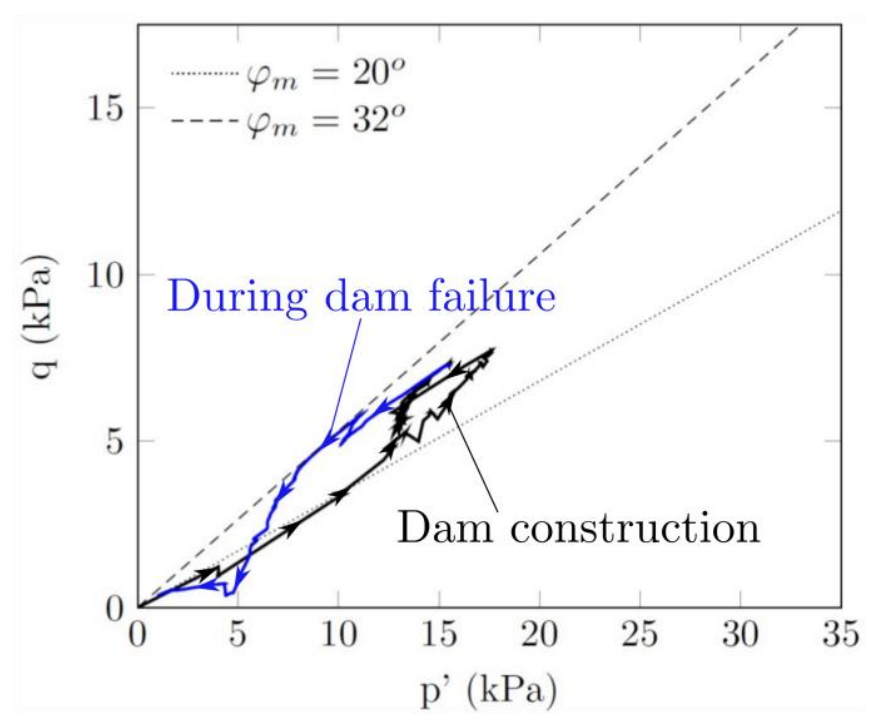

Figure 17. Loading path followed by the material located in zone a (see Figure 10) during the dam construction and the increase of the water height up to $\boldsymbol{H}_{\boldsymbol{w}}=\mathbf{4 . 5} \mathbf{~ m}$ (black line) and after the water height has increased to $\boldsymbol{H}_{\boldsymbol{w}}=\boldsymbol{H}_{\boldsymbol{r}}$ (blue line). The states corresponding to mobilized frictions of $20^{\circ}$ and $32^{\circ}$ are given as dotted and solid lines for the sake of illustration.

In order to better demonstrate the benefits of using the H-model as compared to more standard constitutive models, the numerical simulation was repeated by considering the nonassociated elastoplastic Mohr-Coulomb model with zero dilatancy, as commonly used in engineering practice. The density of the material has been taken to be the same while a friction angle of $32^{\circ}$ has been adopted for the dam material in order to remain consistent with the results displayed in Figure 17. The corresponding FLAC simulation of the dam construction and filling showed that failure is observed for an upstream water level $50 \mathrm{~cm}$ lower than in the previous case. The displacement field and the strain field observed at failure with Mohr-Coulomb constitutive model are shown in Figure 18. 

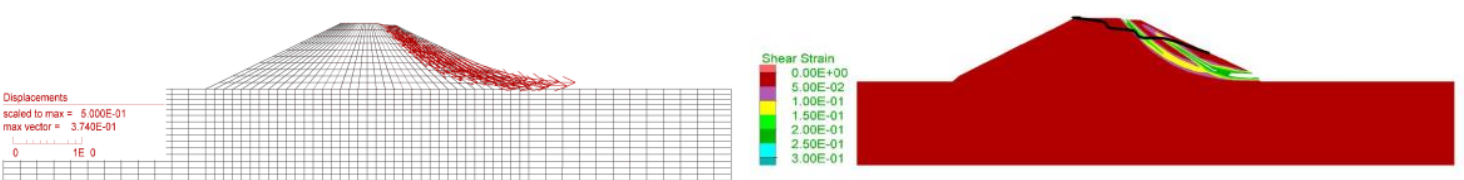

Figure 18. Displacement and deviatoric strain fields obtained with Mohr-Coulomb model

The failure mode corresponds to a conventional circular sliding of the downstream facing crossing the toe of the slope. The comparison between the two simulation cases and the failure patterns of Figure 15 and Figure 18 suggests that the H-model captures the dilatancy more realistically, leading to a higher strength value. For a similar internal friction angle, failure is observed with the H-model at a higher water level. This, together with the more important capability of capturing the evolution of the microstructure, demonstrates the superiority of the H-model over the simple elastoplastic models.

\section{Conclusion}

Inspiring ideas from the multislip theory have been combined with modern multiscale modelling of granular materials to construct the H-model, capable of spanning physics across multiple scales. The developed model represents the granular assembly by a collection of idealized hexagonal (or 3D double-hexagonal) units whose kinematics and statics properties can be readily analyzed at the meso-scale. Further upscaling of these meso-structures results in a macroscopic constitutive model, capable of capturing many salient characteristics of granular media within different deformational regimes.

The eventual model resorts to only 6 material parameters, which are calibrated for Hostun sand. The comparisons with experimental results demonstrate that the model is indeed capable of predicting both stress-strain trends and volumetric responses with an acceptable accuracy. Of note is the model's tendency to underestimate the contraction of both loose and dense samples.

The H-model is next implemented into a FDM solver to study structural scale boundary value problems. FDM simulation of a biaxial test reveals a more realistic depiction of post-peak softening and a quicker approach to the steady state which can be directly related to the inhomogeneities due to shear banding.

Following the validation at the laboratory scale, the FDM model is used to perform a stress analysis for an earth dam as the upstream water level gradually increases. Despite the inhomogeneity of the soil layers and the non-trivial geometry of the domain, the H-model is 
seen to be capable of consistently capturing the typical stress distribution and effective stress dependency of deformation fields.

Moreover, due to its multi-scale nature, the $\mathrm{H}$-model also provides access to micromechanical properties of geomaterials that often fall beyond the reach of continuum models. In this regard, the simulation provides access to the fields of micro- and mesoscopic events such as contact loss and interparticle sliding. As aninteresting new observation, the boundary value problem reveals that, despite what is intuitively expected, the shear failure at the downstream is not associated with the frictional sliding at the particle scale, but rather with opening of cell due to loss of contacts. Such rapid contacts loss events can be associated with the increase of pore water pressure in the dam body (hence the local decrease of effective mean stress) which can eventually lead to liquefaction. Direct comparison with the Mohr-Coulomb elastoplastic model shows how such failure mechanisms falls beyond the reach of simpler constitutive model.

While the detail of such correlations with continuum level behaviors certainly requires more complete analyses, the current study successfully demonstrates the capabilities of H-model in bridging between the particle scale notions on one hand and the structural scale problems on the other. Through the proper incorporation of microstructural state variables into the constitutive model, the $\mathrm{H}$-model provides a method to coherently investigate the macroscopic repercussions of the otherwise inaccessible micromechanical properties such as anisotropy and contact network evolution in a boundary value setting.

The H-model is versatile and it easy to build in new features by merely introducing new physics in an additive manner at the lower scale, a process much more tricky in phenomenological approaches. The nature of the inter-granular contacts can be enriched to address other physics such as capillarity, cohesion or even erosion processes (as illustrated in Figure 3). These ongoing developments will make it possible to account for additional failure mechanisms at the engineering scale. 


\section{Acknowledgements}

The authors wish to thank Na DENG (INRAE) and Qirui MA (Wuhan University) for insightful discussions that helped improve the presentation and detailed discussions around the H-model and its future enriched versions. The authors express their sincere thanks to the French Research Network GeoMech (GDRI CNRS) for promoting vigorous and convivial interactions among the authors of the present paper.

\section{Referencess}

Alshibli, K. A., and Sture, S. (1999). Sand shear band thickness measurements by digital imaging techniques. Journal of computing in civil engineering, 13(2), 103-109.

Balendran, B., and Nemat-Nasser, S. (1993a): Double sliding model cyclic deformation of granular materials including dilatancy effects. Journal of the Mechanics and Physics of Solids, Vol. 41, n³, pp. 573-612.

Balendran, B., and Nemat-Nasser, S. (1993b): Viscoplastic flow of planar granular materials. Mechanics of Materials, Vol. 16, pp. 1-12.

Batdorf, S. B. and Budianski, B. (1949). A mathematical theory of plasticity based on the concept of slip. National Advisory Committee for Aeronautics (N.A.C.A.), Technical Note No. 1871, Washington, DC.

Bathurst, R. J. and Rothenburg, L. (1992). Investigation of micromechanical features of idealized granular assemblies using DEM. Engineering computations.

Bazant, Z.P. (1978): Endochronic inelasticity and incremental plasticity. International Journal of Solids and Structures, Vol. 14, pp. 691-714.

Bazant, Z. P. and Oh, B. H. (1983). Crack band theory for fracture of concrete. Materiaux et Constructions, 16(93), pp. 155-177.

Bazant, Z.P., and Gambarova, P.G. (1984): Crack shear in concrete: crack band microplane model. ASCE Journal of Structural Engineering, Vol. 110, n 9, pp. 2015-2035.

Bazant, Z. P. (1984). Microplane model for strain-controlled inelastic behavior. In Mechanics of Engineering Materials (Edited by C. S. Desai and R. H. Gallagher), Chap. 4, pp. 45-59. John Wiley \& Sons, Chichester and New York.

Bazant, Z. P. and Oh, B. H. (1985). Microplane model for progressive fracture of concrete and rock. J. Engng. Mech., ASCE 111(4), pp. 559-582. 
Christoffersen, J., Mehrabadi, M. M., \& Nemat-Nasser, S. (1981). A micromechanical description of granular material behavior.

De Saxcé, G., Fortin, J., \& Millet, O. (2004). About the numerical simulation of the dynamics of granular media and the definition of the mean stress tensor. Mechanics of Materials, 36(12), 1175-1184.

Darve, F. (1990a): The expression of rheological laws in incremental form and the main classes of constitutive equations. In Geomaterials Constitutive Equations and Modelling, F. Darve ed., Elsevier Applied Science, pp. 123-148.

Darve, F. (1990b): Incrementally non-linear constitutive relationships, in Geomaterials, Constitutive Equations and Modelling, F. Darve ed., Elsevier Applied Science, pp. 213-238

Darve, F., Flavigny, E., and Meghachou, M. (1995): Yield surfaces and principle of superposition revisited by incrementally non-linear constitutive relations. Int. J. Plasticity, Vol. 11, n 8, pp. 927-948.

Darve, F., Servant, G., Laouafa, F., and Khoa H.D.V. (2004): Failure in geomaterials, continuous and discrete analyses. Comp. Methods Appl. Mech. Engrg., Vol. 193, pp. 30573085 .

Desrues, J., Viggiani, G. (2004): Strain localization in sand: an overview of the experimental results obtained in Grenoble using stereo-photogrammetry. International Journal for Numerical and Analytical Methods in Geomechanics, Vol. 28, pp. 279-321.

Drucker, D., and Prager, W. (1952): Soil mechanics and plastic analysis for limit design. Q. Applied Math., Vol. 10, pp. 157-165.

Guo, N., and Zhao, J. (2014): A coupled FEM/DEM approach for hierarchical multiscale modelling of granular media. Int. J. Numer. Meth. Engng., Vol. 99, pp. 789-818.

Hill, R. (1965): Continuum micro-mechanics of elastoplastic polycrystals. J. Mech. Phys. Solids. Vol. 13, pp. 89-101.

Hill, R. (1966): Generalized relations for incremental deformation of metal crystals by multislip. J. Mech. Phys. Solids. Vol. 14, n 2, pp. 95-102.

Hill, R. (1967a): On the classical constitutive relations for elastic-plastic solids. In Recent progress in applied mechanics, Folke Odqvist Volume, B. Broberg, J. Hult and F. Niordson Eds., Almqvist and Wiksell, pp. 241-249.

Hill, R. (1967b): The essential structure of constitutive laws for metal composites and polycrystals. Journal of the Mechanics and Physics of Solids, Vol. 15, n² 2, pp. 79-95. 
Horne, M.R. (1965): The behavior of an assembly of rotund, rigid cohesionless particles - I, II. Proc. Royal Society London, Vol. 286, pp. 62-97.

Kolymbas, D. (1999): Introduction to hypoplasticity. Balkema Publisher, 104 p.

Kolymbas, D. (1991): An outline of hypoplasticity. Archive of Applied Mechanics, Vol. 61, pp. 143-151.

Liu, J., Nicot, F., \& Zhou, W. (2018). Sustainability of internal structures during shear band forming in 2D granular materials. Powder Technology, 338, 458-470.

Liu, J., Wautier, A., Bonelli, S., Nicot, F., \& Darve, F. (2020). Macroscopic softening in granular materials from a mesoscale perspective. International Journal of Solids and Structures, 193, 222-238.

Love, A. E. H. (1892). A treatise on the mathematical theory of elasticity. 1, 1892.

Mehrabadi, M. M., Nemat-Nasser, S., \& Oda, M. (1982). On statistical description of stress and fabric in granular materials. International Journal for Numerical and Analytical Methods in Geomechanics, 6(1), 95-108.

Mehrabadi, M.M., Loret, B., and Nemat-Nasser, S. (1993): Incremental constitutive relations for granular materials based on micromechanics. In Proc. Roy. Soc. London, Vol. 441, pp. 433-463.

Nemat-Nasser, S., and Mehrabadi, M.M. (1984): Micromechanically based rate constitutive descriptions for granular materials. In Mechanics of engineering materials, Proc. Int. Conf. Constitutive Law for Eng. Mat. Theory and application, C.S. Desai and R.H. Gallagher Eds., John Wiley \& Sons, New York.

Nemat-Nasser, S. (2000): A micromechanically-based constitutive model for frictional deformation of granular materials. Journal of the Mechanics and Physics of Solids, Vol. 48, $\mathrm{n}^{\circ}$ 6-7, pp. 1541-1563.

Nemat-Nasser, S., and Zhang, J. (2002): Constitutive relations for cohesionless frictional granular materials. Int. J. of Plasticity, Special issue for Tim Wright, pp. 531-547.

Nicot, F., and Darve, F. (2005): A multiscale approach to granular materials. Mechanics of Materials, Vol. 37 (9), pp. 980-1006.

Nicot, F., and Darve, F. (2007a): Basic features of plastic strains: from micro-mechanics to incrementally nonlinear models. Int. Journal of Plasticity, Vol. 23, pp. 1555-1588.

Nicot, F., and Darve, F. (2007b): Micro-mechanical bases of some salient constitutive features of granular materials. Int. J. of Solids and Structures, Vol. 44, pp. 7420-7443. 
Nicot, F., and Darve, F. (2011a): Diffuse and localized failure modes: two competing mechanisms. International Journal for Numerical and Analytical Methods in Geomechanics, Vol. 35, Issue 5, pp. 586-601.

Nicot, F., and Darve, F. (2011b): The H-microdirectional model: accounting for a mesoscopic scale. Mechanics of Materials, Vol. 43, pp. 918-929.

Nicot, F., Hadda, N., Darve, F. (2013). Second-order work analysis for granular materials using a multiscale approach, International Journal for Numerical and Analytical Methods in Geomechanics, 37, 2987-3007. doi: 10.1002/nag.2175

Oda, M., Nemat-Nasser, S., \& Konishi, J. (1985). Stress-induced anisotropy in granular masses. Soils and foundations, 25(3), 85-97.

Pande, G. N., and Sharma, K. G. (1981). "Implementation of computer procedures and stressstrain laws in geotechnical engineering." Proc., Symp. on Implementation of Compo Procedures and Stress-Strain Laws in Geotech. Engrg., C. S. Desai and S. K. Saxena, eds., Acorn Press, Durham, N.C., pp. 575-590.

Pande, G. N., and Sharma, K. G. (1982). "Multi-laminate model of clays-A numerical evaluation of the influence of rotation of the principal stress axis." Rep., Dept. of Civ. Engrg., Univ. ColI. of Swansea, U.K.

Pastor, M., Zienkiewicz, O.C., and Chan, A.H.C. (1990): Generalized plasticity and the modeling on soil behavior. Int. Journal for Numerical and Analytical Methods in Geomechanics, Vol. 14, pp. 151-190.

Peters, J. F., Muthuswamy, M., Wibowo, J., Tordesillas, A. (2005): Characterization of force chains in granular material. Physical review E, 72(4), 041307.

Pouragha, M., Wan, R. (2016). Onset of structural evolution in granular materials as a redundancy problem. Granular Matter, 18(3), pp. 38.

Pouragha, M., Wan, R. (2017): Non-dissipative structural evolutions in granular materials within the small strain range. International Journal of Solids and Structures, 110, pp. 94-105.

Pouragha, M., Wan, R. (2018a): On elastic deformations and decomposition of strain in granular media. International journal of solids and structures, 138, pp. 97-108.

Pouragha, M., Wan, R. (2018b): $\mu$-GM: a purely micromechanical constitutive model for granular materials. Mechanics of Materials, 126, pp. 57-74.

Pouragha, M., Duriez, J., Wautier, A., Wan, R., Nicot, F., Darve, F. (2019): Preferential growth of force network in granular media. Granular Matter, 21(3), pp. 67. 
Radjai, F., Wolf, D., Jean, M., and Moreau, J.J. (1998): Bimodal character of stress transmission in granular packing. Physical Review Letters, Vol. 80, n 1, pp. 61-64.

Radjai, F., Roux, S., and Moreau, J.J. (1999): Contact forces in a granular packing. Chaos, Vol. $9, \mathrm{n}^{\circ} 3$, pp. 544-550.

Rice, J.R. (1970): On the structure of stress-strain relations for time-dependent plastic deformation in metals. Journal of Applied Mechanics, Vol. 37, pp. 728-737.

Rice, J.R. (1975): Continuum mechanics and thermodynamics of plasticity in relation to microscale deformation mechanisms. Constitutive equations in plasticity, A.S. Argon Ed., MIT Press, Cambridge, pp. 23-79.

Saada, A.S. (1989): Constitutive Equations for Granular Soils, Balkema publ.

Sterpi, D. (1999). An analysis of geotechnical problems involving strain softening effects. International Journal for Numerical and Analytical Methods in Geomechanics, 23(13), 1427 1454.

Taylor, G.I. (1934): The mechanism of plastic deformation of crystals - I, theoretical. Proc. Of the Royal Society of London, A, Vol. 145, pp. 362-387.

Taylor, G.I. (1938): Plastic strains in metals. J. Inst. Metals, Vol. 62, pp. 307-325.

Tordesillas, A. (2007): Force chain buckling, unjamming transitions and shear banding in dense granular assemblies. Phil. Mag. Vol. 87(32), pp. 4987-5016.

Tordesillas, A., and Muthuswamy, M. (2009): On the modeling of confined buckling of force chains. Journal of the Mechanics and Physics of Solids, Volume 57(4), pp. 706-727.

Tordesillas, A., Walker, D.M., and Lin, Q. (2010): Force cycles and force chains. Physical Review E, Vol. 81, 011302.

Veylon, G., Nicot, F., Zhu, H., Darve, F. (2018). Microstructure Incidence on the Bifurcation Domain Topology in Granular Materials, Journal of Engineering Mechanics, 144(6), 04018031. doi: 10.1061/(ASCE)EM.1943-7889.0001446.

Walker, D.M., and Tordesillas, A. (2010): Topological evolution in dense granular materials: a complex networks perspective. Int. J. of Solids and Structures, Vol. 47, pp. 624-639.

Wautier, A., Bonelli, S., and Nicot, F. (2017). Scale separation between grain detachment and grain transport in granular media subjected to an internal flow. Granular Matter, 19(2), 22.

Wautier, A., Bonelli, S., and Nicot, F. (2019). Rattlers' contribution to granular plasticity and mechanical stability. International Journal of Plasticity, 112, 172-193. 
Xiong, H., Yin, Z. Y., and Nicot, F. (2018): A multiscale second-order work analysis approach for geotechnical structures. Int. J. for Numerical and Analytical Methods in Geomechanics, 10.1002/nag.2893.

Xiong, H., Miot, M., Veylon, G., Wautier, A., Philippe, P., Yin, Z. Y., Nicot, F. and Darve, F. (2020): A novel multi-scale large deformation approach for modelling of granular collapse. Acta Geotechnica. In press.

Zienkiewicz, O. C., and G. N. Pande (1977): Time dependent multilaminate model of rocks, a numerical study of deformation and failure of rock masses. International Journal for Numerical and Analytical Methods in Geomechanics, Vol. 1, pp. 219-247.

Zienkiewicz, O.C., and Mroz, Z. (1984): Generalized plasticity formulation and applications to geomechanics. Mechanics of Engineering Materials, C.S. Desai and R.H. Gallagher Eds., Wiley, 655-679.

Zhu H., Nicot F., Darve F. (2016a): Meso-structure evolution in a 2D granular material during biaxial loading, Granular Matter, 18(1), pp. 1-12

Zhu H., Nicot F., Darve F. (2016b): Meso-structure organization in two-dimensional granular materials along biaxial loading paths, Int. J. Solids and Struct., 96, pp. 25-37 


\section{Appendix: Detailed review of the H-model}

\section{A. Constitutive behavior of a unit H-Cell}

In the H-model, the microstructure of granular materials is assumed to be described by a collection of hexagonal cells in 2D composed of grains idealized as disks of equal radii and distributed in space with different orientations. Figure 2 illustrates one of these cells in static equilibrium under the action of external forces.

As shown in Figure 2, each H-cell is described by only three geometrical parameters: the opening angle $\alpha$, and the inter-granular distances $d_{1}$ and $d_{2}$. These relate to the unit cell dimensions $l_{1}$ and $l_{2}$ as

$$
\left\{\begin{array}{c}
l_{1}=d_{2}+2 d_{1} \cos \alpha \\
l_{2}=2 d_{1} \sin \alpha
\end{array}\right.
$$

For dry frictional granular materials, the local mechanical behavior is an elastic-frictional contact law. Such a contact law relies on three parameters: a normal stiffness $k_{n}$, a tangential stiffness $k_{t}$, and an inter-granular friction angle $\varphi_{g}$. If a contact $i$ persists during an incremental evolution, the normal and tangential contact forces can be expressed in incremental form as

$$
\left\{\begin{aligned}
& \delta N_{i}= k_{n} \delta u_{n}^{i} \\
& \delta T_{i}= \begin{cases}T_{i}+k_{t} \delta u_{t}^{i}, & \text { if }\left|T_{i}+k_{t} \delta u_{t}^{i}\right|<\tan \varphi_{g}\left(N_{i}+\delta N_{i}\right) \\
\xi \tan \varphi_{g}\left(N_{i}+\delta N_{i}\right)-T_{i} & \text { if }\left|T_{i}+k_{t} \delta u_{t}^{i}\right| \geq \tan \varphi_{g}\left(N_{i}+\delta N_{i}\right)\end{cases}
\end{aligned}\right.
$$

where $\xi$ is the sign of $T_{i}$, and $u_{n}^{i}$ and $u_{t}^{i}$ are the normal and tangential relative displacements of the grains at contact $i$. Given the symmetries in the H-cell, we only need to consider the contact forces $N_{1}, N_{2}$ and $T_{1}$, and the corresponding incremental displacements are obtained by differentiating the expression of the branch vector joining the grains in contact:

$$
\left\{\begin{array}{l}
\delta u_{n}^{1}=-\delta d_{1} \\
\delta u_{n}^{2}=-\delta d_{2} \\
\delta u_{t}^{1}=d_{1} \delta \alpha
\end{array}\right.
$$

As a result, the incremental evolutions of the contact forces are given as

$840 \quad\left\{\begin{array}{r}\delta N_{1}=-k_{n} \delta d_{1} \\ \delta N_{2}=-k_{n} \delta d_{2}\end{array} \quad \begin{array}{l}\text { if }\left|T_{1}+k_{t} d_{1} \delta \alpha\right|<\tan \varphi_{g}\left(N_{1}-k_{n} \delta d_{1}\right) \\ \delta T_{1}= \begin{cases}T_{1}+k_{t} d_{1} \delta \alpha, & \text { if }\left|T_{1}+k_{t} d_{1} \delta \alpha\right| \geq \tan \varphi_{g}\left(N_{1}-k_{n} \delta d_{1}\right) \\ \xi \tan \varphi_{g}\left(N_{1}-k_{n} \delta d_{1}\right)-T_{1}\end{cases} \end{array}\right.$ 
where $\xi$ is the sign of $T_{1}$.

By differentiating equation (A.1), the incremental evolutions $\delta l_{1}$ and $\delta l_{2}$ can be related to $\delta d_{1}, \delta d_{2}$ and $\delta \alpha$ (geometrical compatibility). It should be noted that the relative evolutions of

In the end, the incremental evolutions $\delta l_{1}$ and $\delta l_{2}$ can be conveniently related to $\delta d_{1}, \delta d_{2}$ and $\delta \alpha$ in matrix form as

Depending on the expression for $\delta T_{1}$, the compatibility equation reads:

$$
\left\{\begin{array}{rr}
k_{n} \cos \alpha \delta d_{1}-k_{n} \delta d_{2}+\left[N_{1} \sin \alpha-T_{1} \cos \alpha-k_{t} d_{1}(1+\sin \alpha)\right] \delta \alpha=0 \\
\text { (if no sliding occurs) } \\
{\left[k_{n} \cos \alpha \delta d_{1}+\xi k_{n} \tan \varphi_{g}(1+\sin \alpha)\right] \delta d_{1}-k_{n} \delta d_{2}+\left[N_{1} \sin \alpha-T_{1} \cos \alpha\right] \delta \alpha=} \\
\xi(1+\sin \alpha)\left(\tan \varphi_{g} N_{1}-T_{1}\right) & \text { (if sliding occurs) }
\end{array}\right.
$$

$$
\boldsymbol{A}\left[\begin{array}{c}
\delta d_{1} \\
\delta d_{2} \\
\delta \alpha
\end{array}\right]=\left[\begin{array}{c}
\delta l_{1} \\
\delta l_{2} \\
\lambda
\end{array}\right]
$$

where $\lambda$ is non-zero only when sliding activates $\left(\lambda=\xi(1+\sin \alpha)\left(\tan \varphi_{g} N_{1}-T_{1}\right)\right)$. This relationship completes the constitutive behavior of the unit $\mathrm{H}$-cell by relating the geometric evolution $\left(\delta l_{1}, \delta l_{2}\right)$ to the contact force evolutions $\left(\delta N_{1}, \delta N_{2}, \delta T_{1}\right)$.

\section{B. Homogenization scheme}

The objective is to arrive by way of directional homogenization at a stress-strain relationship for a REV endowed with a microstructure that consists of a collection of unit $\mathrm{H}$-cells distributed in space with different orientations. 
The general homogenization scheme of the H-model starts from the macroscale to zoom into the mesoscale, and to thereafter upscale back to the macroscale following the strategy proposed in Figure 3.

The first stage of the homogenization scheme is known as the kinematic localization hypothesis (inherited from the microdirectional model (Nicot and Darve (2005)). It assumes that any macroscopic incremental strain $\boldsymbol{\delta} \boldsymbol{\varepsilon}$ is transferred to all contacts of the H-cells at the lower scale (i.e. homogeneous strain). By introducing the local frame $(\boldsymbol{n}, \boldsymbol{t})$ of a given unit $\mathrm{H}-$ cell of dimensions $\left(l_{1}, l_{2}\right)$, kinematic localization translates into:

$$
\left\{\begin{array}{l}
\frac{\delta l_{1}}{l_{1}}=\boldsymbol{n}^{\boldsymbol{T}} \cdot \boldsymbol{\delta} \boldsymbol{\varepsilon} \cdot \boldsymbol{n} \\
\frac{\delta l_{2}}{l_{2}}=\boldsymbol{t}^{\boldsymbol{T}} \cdot \boldsymbol{\delta} \boldsymbol{\varepsilon} \cdot \boldsymbol{t}
\end{array}\right.
$$

where $\boldsymbol{n}^{\boldsymbol{T}}$ stands for the transpose of vector $\boldsymbol{n}$. In the 2D case, the orientation of the unit H-cell in the global frame is characterized by a single angle $\theta \in[0, \pi]$ such that $\boldsymbol{n}=\left[\begin{array}{ll}\cos \theta & \sin \theta\end{array}\right]^{T}$ and $\boldsymbol{t}=\left[\begin{array}{ll}-\sin \theta & \cos \theta\end{array}\right]^{T}$.

Subsequent stages 2, 3 and 4 in Figure 3 involve applying the constitutive equation of the unit H-cell that was worked out in the previous subsection. Finally, in the last stage, the macroscopic stress tensor $\boldsymbol{\sigma}$ of the REV is calculated by a directional averaging of all contact forces acting in a collection of unit H-cells. This is done in two steps by first defining a mesostress $\boldsymbol{\sigma}_{\text {meso }}$ for each $\mathrm{H}$-cell and then averaging all the meso-stresses of the H-cell collection.

Here and below, the meso-stress tensor is defined at the scale of the unit H-cell using the Love-Weber formula (Love, 1892; Christoffersen et al., 1981; Mehrabadi et al., 1982; De Saxcé et al., 2004):

$$
\boldsymbol{\sigma}_{\text {meso }}=\frac{1}{V_{\text {meso }}} \sum_{c \in V_{\text {meso }}} \boldsymbol{f}_{c} \otimes \boldsymbol{l}_{c}
$$

where $\boldsymbol{f}_{\boldsymbol{c}}$ is the contact force at contact $c, \boldsymbol{l}_{\boldsymbol{c}}$ the branch vector joining the centers of the two grains in contact and $V_{\text {meso }}=l_{1} l_{2}$ the volume of the unit H-cell.

Expressed in the local frame (n,t), equation (A.8) gives with soil mechanics conventions (positive compressions)

$$
\left\{\begin{array}{c}
V_{\text {meso }} \sigma_{n n}^{\text {meso }}=4 N_{1} d_{1} \cos ^{2} \alpha+4 T_{1} d_{1} \cos \alpha \sin \alpha+2 N_{2} d_{2} \\
V_{\text {meso }} \sigma_{t t}^{\text {meso }}=4 N_{1} d_{1} \sin ^{2} \alpha-4 T_{1} d_{1} \cos \alpha \sin \alpha \\
V_{\text {meso }} \sigma_{n t}^{\text {meso }}=V_{\text {meso }} \sigma_{\text {tn }}^{\text {meso }}=0
\end{array}\right.
$$


It should be noted that equation (A.9) assumes that contacts 1 and 2 exist $\left(N_{1}>0\right.$ and $\left.N_{2}>0\right)$, and that grain pairs 2-6, 3-5 and 1-4 in Figure 2 are not in contact. In case, contact 1 or 2 is lost $\left(d_{1}>D\right.$ or $\left.d_{2}>D\right)$, the meso-stress is set to $\boldsymbol{\sigma}_{\text {meso }}=\mathbf{0}$ instead. In case additional contacts create, additional terms have to be accounted for in equation (A.8). Such terms contribute to $\sigma_{n n}^{m e s o}$ if contact 1-4 forms or to $\sigma_{t t}^{m e s o}$ if contacts 2-6 and 3-5 forms. The meso-stress computed from equation (A.9) is then updated with the following correction term

$$
V_{\text {meso }} \boldsymbol{\sigma}_{\text {corr }}^{\text {meso }}=\left\{\begin{array}{cc}
k_{n}\left(D-l_{1}\right) l_{1} \boldsymbol{n} \otimes \boldsymbol{n} & \text { if } l_{1}<D \\
2 k_{n}\left(D-l_{2}\right) l_{2} \boldsymbol{t} \otimes \boldsymbol{t} & \text { if } l_{2}<D
\end{array}\right.
$$

Finally, the macroscopic stress tensor for the REV can be determined as an integration of all meso-stresses of individual unit H-cells, knowing their statistical distribution via the probability density function $\omega(\theta)$. Thus, for the entire collection of unit H-cells,

$$
\boldsymbol{\sigma}=\frac{1}{V} \int_{\theta=0}^{\pi} \omega(\theta) V_{\text {meso }}(\theta) \boldsymbol{\sigma}_{\text {meso }}(\theta) \mathrm{d} \theta
$$

such that

$$
V=\int_{\theta=0}^{\pi} \omega(\theta) V_{\text {meso }}(\theta) \mathrm{d} \theta \text { and } \int_{\theta=0}^{\pi} \omega(\theta) \mathrm{d} \theta=1 .
$$

If $\boldsymbol{\sigma}_{\text {meso }}$ is expressed as a matrix in the local frame $(\boldsymbol{n}, \boldsymbol{t})$, the stress matrix $\boldsymbol{\sigma}$ in the global frame $\left(\boldsymbol{e}_{1}, \boldsymbol{e}_{2}\right)$ is readily obtained by conversion from local to global configurations, i.e.

$$
\boldsymbol{\sigma}^{\left(\boldsymbol{e}_{1}, \boldsymbol{e}_{2}\right)}=\frac{1}{V} \int_{\theta=0}^{\pi} \omega(\theta) V_{\text {meso }}(\theta) \boldsymbol{P}^{-1} \boldsymbol{\sigma}_{\text {meso }}^{(\boldsymbol{n}, \boldsymbol{t})}(\theta) \boldsymbol{P} \mathrm{d} \theta
$$

where $\boldsymbol{P}$ is the transformation matrix, i.e.

$$
\boldsymbol{P}=\left(\begin{array}{cc}
\cos \theta & \sin \theta \\
-\sin \theta & \cos \theta
\end{array}\right)
$$

It is noteworthy that the volumetric change of the meso-volume chosen as the bounding box of the inner hexagonal loop $\left(V_{\text {meso }}=l_{1} l_{2}\right)$ leads to the trace of $\boldsymbol{\delta} \boldsymbol{\varepsilon}$ when using the localizationhypothesis, i.e.

$$
\frac{\delta V_{\text {meso }}}{V_{\text {meso }}}=\frac{\delta l_{1}}{l_{1}}+\frac{\delta l_{2}}{l_{2}}=\operatorname{Tr}(\boldsymbol{\delta} \boldsymbol{\varepsilon})
$$

Statistical averaging then evidently gives $\delta V=\int_{\theta=0}^{\pi} \omega(\theta) \delta V_{\text {meso }}(\theta) \mathrm{d} \theta=\operatorname{Tr}(\boldsymbol{\delta} \boldsymbol{\varepsilon}) V$, which is consistent with the classical interpretation of $\operatorname{Tr}(\boldsymbol{\delta} \boldsymbol{\varepsilon})$ at macroscale. 
While $V_{\text {meso }}$ is selected here as the bounding box of an inner hexagonal loop, it should be underlined that the choice of $V_{\text {meso }}$ is not unique as long as the strain localization equation (A.7) is updated accordingly to ensure that $\frac{\delta V_{\text {meso }}}{V_{\text {meso }}}=\operatorname{Tr}(\boldsymbol{\delta} \boldsymbol{\varepsilon})$. 